\title{
Quark-nova remnants
}

\section{Application to radio emitting anomalous X-ray pulsars transients}

\author{
R. Ouyed, D. Leahy, and B. Niebergal
}

\author{
Department of Physics and Astronomy, University of Calgary, 2500 University Drive NW, Calgary, Alberta, T2N 1N4, Canada \\ e-mail: ouyed@phas.ucalgary.ca
}

Received 9 October 2008 / Accepted 12 April 2010

\begin{abstract}
XTE J1810-197 and 1E 1547.0-5408 are two transient anomalous X-rays pulsars (AXPs) exhibiting radio emission with unusual properties. In addition, their spin down rates during outburst show opposite trends, which so far has no explanation. Here, we extend our quark-nova model for AXPs to include transient AXPs, in which the outbursts are caused by transient accretion events from a Keplerian (iron-rich) degenerate ring. For a ring with inner and outer radii of $23.5 \mathrm{~km}$ and $26.5 \mathrm{~km}$, respectively, our model gives a good fit to the observed X-ray outburst from XTE J1810-197 and the behavior of temperature, luminosity, and area of the two X-ray blackbodies with time. The two blackbodies in our model are related to a heat front (i.e. Bohm diffusion front) propagating along the ring's surface and an accretion hot spot on the quark star surface. Radio pulsations in our model are caused by dissipation at the light cylinder of magnetic bubbles, produced near the ring during the X-ray outburst. The delay between X-ray peak emission and radio emission in our model is related to the propagation time of these bubbles to the light cylinder and scale with the period as $t_{\text {prop. }} \propto P^{\frac{7}{2}-\frac{\alpha}{2}} / \dot{P}^{1 / 2}$ where $\alpha$ defines the radial dependence of matter density in the magnetosphere $\left(\propto r^{-\alpha}\right)$; for an equatorial wind, $\alpha=1$, we predict a $\sim 1$ year and $\sim 1$ month delay for XTE J1810-197 and 1E 1547.0-5408, respectively. The observed flat spectrum, erratic pulse profile, and the pulse duration are all explained in our model as a result of X-point reconnection events induced by the dissipation of the bubbles at the light cylinder. The spin down rate of the central quark star can either increase or decrease depending on how the radial drift velocity of the magnetic islands changes with distance from the central star. We suggest an evolutionary connection between transient AXPs and typical AXPs in our model.
\end{abstract}

Key words. stars: evolution - accretion, accretion disks - magnetic fields - pulsars: general

\section{Introduction}

Anomalous X-ray pulsars (AXPs) are magnetars with rotation period of 2-12 s and inferred surface magnetic field strength $B \sim 10^{14-15} \mathrm{G}$ (e.g. Woods \& Thompson 2006; Kaspi 2007). In this work we focus on 2 AXPs, XTE J1810-197 and 1E 1547.0-5408, which are the only magnetars known to emit in the radio (Camilo et al. 2006). Both are demonstrably transient radio sources, having not been detected in previous surveys of adequate sensitivity. XTE J1810-197 is a transient AXP $^{1}$ first detected when its X-ray flux increased $\sim 100$-fold compared to a quiescent level it maintained for at least 24 years (Ibrahim et al. 2004). Discovered with the Einstein X-ray satellite in 1980, 1E 1547.0-5408 was eventually identified as a magnetar candidate (Gelfand \& Gaensler 2007) with spectral characteristics of an AXP. In this paper we look at these sources in the Quark-Nova context (hereafter QN; Ouyed et al. 2002) building on three previous papers where we explore its application to Soft Gammaray Repeaters (SGRs) (Ouyed et al. 2007a; OLNI), to AXPs (Ouyed et al. 2007b; OLNII), and to Rotating Radio Transients (RRATs) (Ouyed et al. 2009; OLNIII), and superluminous supernovae (Leahy \& Ouyed 2008). But first, we briefly describe their observed X-ray and radio properties, during quiescence and bursting phases.

\footnotetext{
${ }^{1}$ In the sense that in quiescence their surface temperature are as low as those of some ordinary young neutron stars.
}

\subsection{The $X$-ray emission}

In the pre-burst era, XTE J1810-197's ROSAT spectrum showed a single blackbody (BB) with temperature $T=0.18 \mathrm{keV}$, an emitting area of $\sim 520 \mathrm{~km}^{2}(d / 3.3 \mathrm{kpc})^{2}$, and a luminosity of $L_{\mathrm{BB}} \sim 5.6 \times 10^{33} \mathrm{erg} \mathrm{s}^{-1}(d / 3.3 \mathrm{kpc})^{2}$. During its bursting phase, XTE J1810-197 showed a hot blackbody ( $T \sim 0.65 \mathrm{keV})$ with an exponential decay in X-ray luminosity of $\sim 280$ days, as well as a warm blackbody $(T \sim 0.3 \mathrm{keV})$ decaying at a rate of $\sim 870$ days (Gotthelf \& Halpern 2007). For the case of 1E 1547.0-5408, after its radio detection (Camilo et al. 2007a), an X-ray outburst was confirmed (Halpern et al. 2008) with a record high luminosity of $\sim 1.7 \times 10^{35}(d / 9.9 \mathrm{kpc})^{2} \mathrm{erg} \mathrm{s}^{-1}$ and with a total outburst energy of $10^{42} \mathrm{erg}<E_{\mathrm{b}}<10^{43} \mathrm{erg}$.

\subsection{The radio emission}

For XTE J1810-197, the radio emission began within $1 \mathrm{yr}$ of its only known X-ray outburst (Camilo et al. 2006, and references therein). At its observed peak more than $3 \mathrm{yr}$ after the X-ray outburst, the radio flux density was more than 50 times the pre-burst upper limit. The X-ray flux has since returned to its quiescent level nearly 4 yrs after the burst. 1E 1547.0-5408, although not as well sampled as XTE J1810-197, exhibits similar variations in flux density and was reported with a factor of 16 times the pre-burst upper limit (Camilo et al. 2007a). 

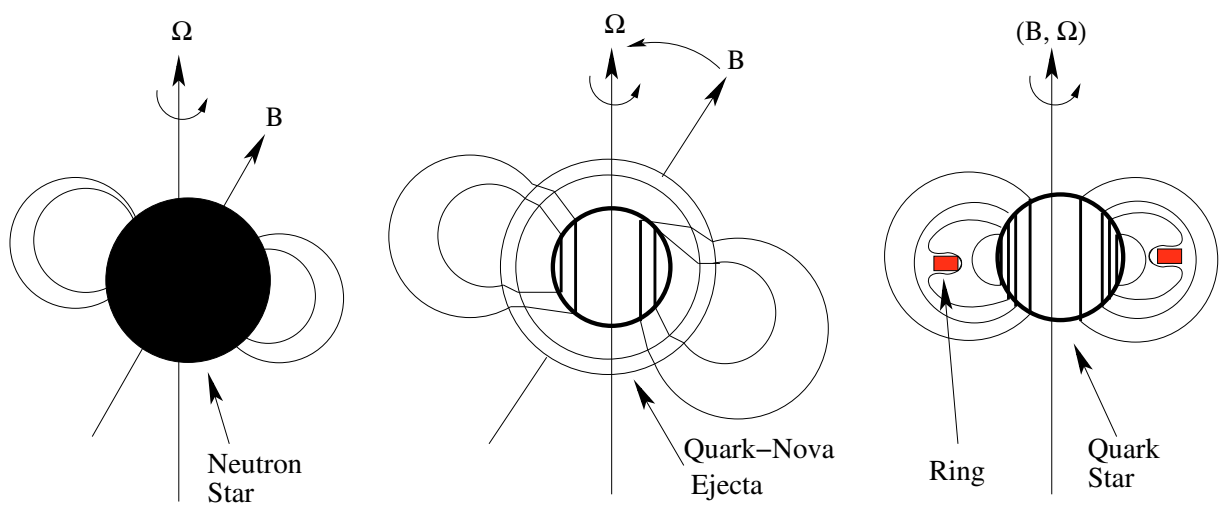

Trends in radio emission between the 2 sources can be summarized as follows:

- both are very highly linearly polarized showing a flat spectrum over a wide range of frequencies. Their striking spectra (i.e. spectral index $>-0.5$ ) clearly distinguishable from ordinary radio pulsars (with a spectral index $\sim-1.6$; Camilo et al. 2007a,b);

- at their peak, both magnetars are very luminous in radio with luminosity at $1.4 \mathrm{GHz} L_{1.4} \geq 100 \mathrm{mJy}(d / \mathrm{kpc})^{2}$, which is larger than the $L_{1.4} \leq 2 \mathrm{mJy}(d / \mathrm{kpc})^{2}$ of most any ordinary young pulsar (e.g. Camilo et al. 2006). For XTE J1810-197, its assumed isotropic radio luminosity up to $42 \mathrm{GHz}$ is about $2 \times 10^{30} \mathrm{erg} \mathrm{s}^{-1}$ (Camilo et al. 2006);

- both have variable pulse profiles (exhibiting sudden changes in radio pulse shape) and radio flux densities. The flux changes at all frequencies. At a given frequency there is no stable average pulse profile. Different pulse components change in relative intensity and new components sometimes appear. Sub-pulses with typical width approximately $<10 \mathrm{~ms}$ are observed (Camilo et al. 2007a,b);

- for XTE J1810-197, the torque was decreasing, at a time when the star was returning to quiescence years after the large outburst. As the torque decreased, so did the radio flux (Camilo et al. 2007b);

- in 1E 1547.0-5408, in contrast, the torque has been increasing, at a time when the X-ray flux has been gradually decreasing (Camilo et al. 2007a).

In this paper we extend our existing quark star model for AXPs to account for the observed behavior of these two transients. This paper is structured as follows: Sect. 2 gives the basic elements of the model. Section 3 describes the quiescent phase. Section 4 , the bursting phase. Section 5, the radio emission. Model predictions are highlighted in Sect. 6 before we conclude in Sect. 7.

\section{Basic components of the model}

The Quark-Nova is an explosive transition from a neutron star (NS) to quark star (QS) (Ouyed et al. 2002; Keränen \& Ouyed 2003). The result is a partial ejection of the NS crust (Keränen et al. 2005) that leads to two possible types of debris surrounding the compact remnant (i.e. the QS) depending on the QS's birth period. In OLNI, we showed that if the QS is born slowly rotating, then the debris formed from the QN ejecta will be in co-rotation, which we argue is responsible for SGRs. In OLNII, we showed that for QS born with millisecond periods,

\subsection{The Keplerian ring}

Fig. 1. Illustrated here are the stages involved in the transition from a neutron star (NS) (inclined rotator) to a quark star (QS) (aligned rotator) in the Quark-Nova scenario. The collapse of the quark core induces an explosive NS-to-QS transition ejecting iron-rich degenerate crust material. The QS enters a superconductive phase confining the interior field to vortices, forcing the exterior field to align with the rotation axis (see Ouyed et al. 2004, for more details; and Ouyed et al. 2006, for the related simulations). The iron-rich degenerate ejecta evolves into a Keplerian ring (at about $15-30 \mathrm{~km}$ from the star) surrounded, but not penetrated, by the dipole field (a co-rotating ejecta is also possible depending on the NS period; see OLNI).

the debris evolves into a Keplerian ring with applications to AXPs. Furthermore, RRATs are the result of late evolution of the Keplerian ring in our model (OLNIII). The interested reader is referred to these papers for more details. Below we give an overview of the salient features of the model in the case of a Keplerian ring before we apply our model to transient AXPs.

\subsection{The quark star: magnetically aligned rotator}

The QN compact remnant is a quark star in the Color-FlavorLocked (CFL) phase, which due to it's rigorously electric neutrality (Rajagopal \& Wilczek 2001) possesses no crust. Owing to the superconductivity of the CFL state, the star's interior contains a lattice of vortices that confine the magnetic field (Ouyed et al. 2004). This interior configuration consequently forces the exterior field to align with the rotation axis (Ouyed et al. 2006; Niebergal et al. 2006); this is illustrated in Fig. 1.

Here we are concerned with compact remnants born with millisecond periods. As shown in OLNII, the quark star is surrounded by Keplerian debris we refer to as a ring. This Keplerian ring is described in detail in Sect. 2.1 and Appendix A in OLNII. Briefly, it is a high density $\left(>\sim 10^{8} \mathrm{~g} \mathrm{~cm}^{-3}\right)$ ring rich in irongroup degenerate material (the ejected NS crust material) in Keplerian rotation around the QS. The ring is highly conducting, cool, and not threaded by the magnetic field. It is a (possibly amorphous) crystalline solid (like the outer crust of a neutron star). The ring expands vertically and radially in time to a structure depicted in Fig. 2 with the ring inner radius at $R_{\text {in }} \sim 15 \mathrm{~km}$, and an outer radius at $R_{\text {out }} \sim 25 \mathrm{~km}$ (for these fiducial values the total area of the ring is $A_{\text {ring }} \sim 3000 \mathrm{~km}^{2}$ ).

The ring thickness in the $z$-direction can be shown to be $H_{\text {ring }} \sim 2.68 \mathrm{~km} \rho_{\text {ring, } 9}^{1 / 6} R_{15}^{3 / 2}$ where $\rho_{\text {ring, } 9}$ is the ring's density in units of $10^{9} \mathrm{~g} \mathrm{~m} \mathrm{~cm}^{-3}$. In reality, on finer scales than depicted in Fig. 2, the ring is subject to tidal fracture and is made up of many cylinders we refer to as "walls". The width of each cylinder is set by Keplerian shear resulting in meter size pieces. The mass and width of a wall are given in OLNII and are $m_{\mathrm{w}} \sim 10^{-10} M_{\odot}$ and $\delta r_{\mathrm{w}} \sim 400 \mathrm{~cm} R_{\mathrm{in}, 15}^{3 / 2}$, respectively.

The magnetic field penetrates a conductor of thickness $\delta r$ on timescale $\tau_{\mathrm{B}} \sim\left(4 \pi \sigma / c^{2}\right) \times(\delta r)^{2}$ where $\sigma$ is the conductivity (e.g. Sect. 4.1 in OLNII). The QS dipole field will penetrate the ring via its innermost wall. The wall is penetrated radially because $\delta r_{\mathrm{w}} \ll H_{\text {ring }}$. This occurs on timescales of a few hundred years 
R. Ouyed et al.: Quark-nova and radio-emitting AXP transients. IV.

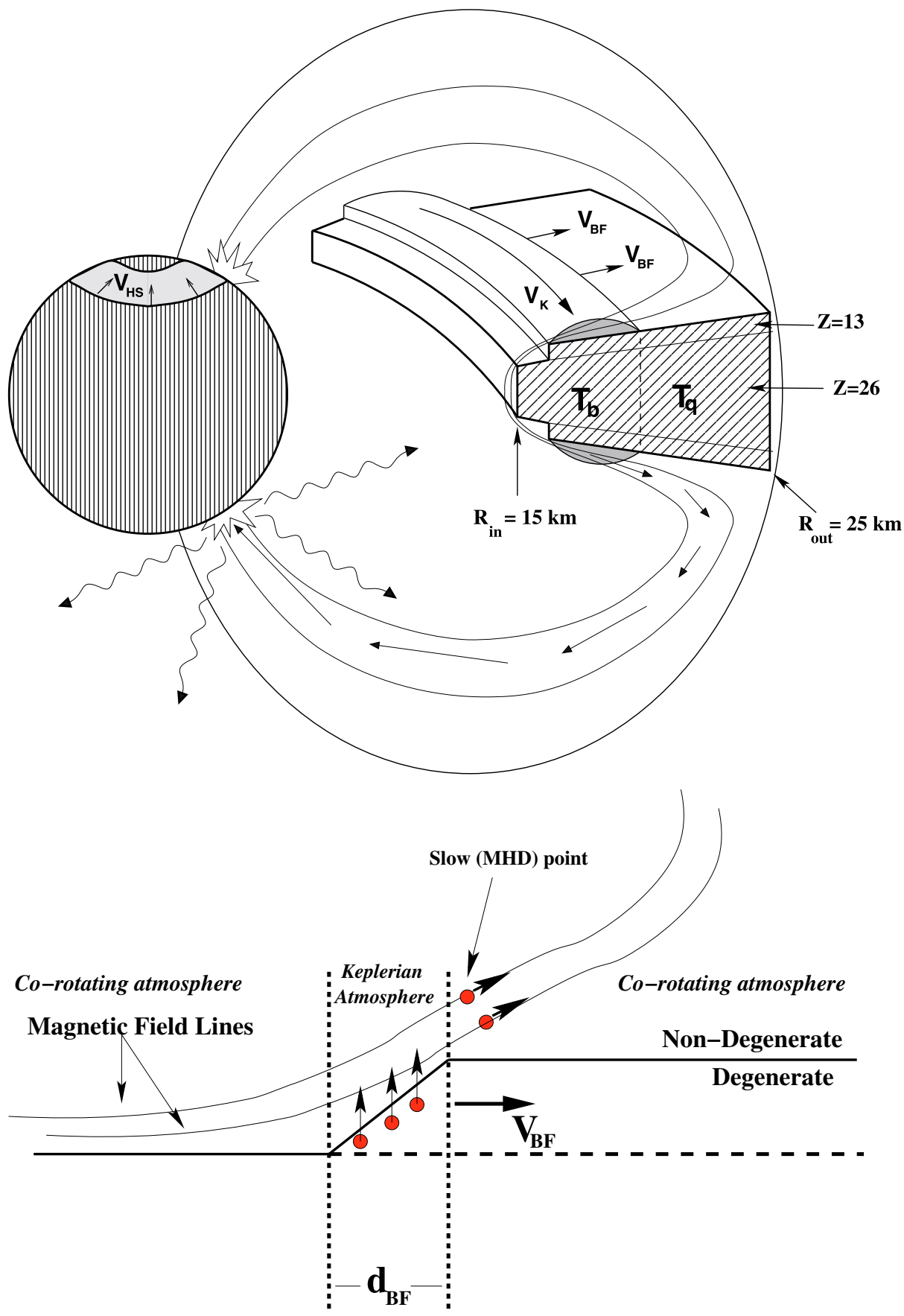

Fig. 2. The upper panel illustrates the ring structure during outburst. Fiducial values of the ring inner radius $(15 \mathrm{~km})$ and outer radius $(25 \mathrm{~km})$ are shown; the ring's vertical scale height is a few kilometers (see Sect. 2.2; the figure is not to scale). The ring is surrounded, but not penetrated, by the dipole field. The Bohm Front - "bump" made of non-degenerate Keplerian material - propagates outward at a speed $V_{\mathrm{BF}}$ heating up the system from $T_{\mathrm{q}}$ to $T_{\mathrm{b}}$ and inducing accretion onto the star. The quark star (an aligned rotator) is also shown with the accretion hot spot shrinking towards the pole at a speed $V_{\mathrm{HS}}$ as a response to the outward motion of the accreting Bohm front. The lower panel illustrates motion and flow dynamics in the vicinity of the Bohm Front (BF). The solid line represents the transition to non-degenerate densities in the ring, $\rho_{\text {nd }}$, while $d_{\mathrm{BF}}$ is the radial width of the BF. As non-degenerate Keplerian material is expelled from the slow point, more Keplerian material is supplied from underneath (see Appendix B). Accretion shuts off once the magnetic field penetrates the nondegenerate Keplerian layer re-enforcing corotation (behind the $\mathrm{BF}$ ).

(see Eq. (17) in OLNII). As the QS magnetic field penetrates the innermost wall, magnetic torques (due to induced $B_{\phi}$ ) slow down the wall, so it can accrete (see Sect. 4).

\subsection{The ring atmosphere}

The ring's density decreases with height above the equatorial plane. At any given temperature the ring's density below which the ring's matter becomes non-degenerate is found by equating the ring temperature to its Fermi temperature; this defines the ring's vertical atmosphere. The atmosphere is characterized by its base density, scale height in the z-direction (vertical to the orbital plane), scale height in the radial direction (i.e. on the outer ring edge, $R_{\text {out }}$; see Appendix B in OLNII), and thermal speed,

$$
\begin{aligned}
& \rho_{\mathrm{atm}} \simeq 460 \mathrm{~g} \mathrm{~m} \mathrm{~cm}^{-3} T_{\mathrm{keV}}^{3 / 2} \\
& H_{\mathrm{atm}}^{z} \simeq 20.4 \mathrm{~cm} \frac{T_{\mathrm{keV}} R_{15}^{3 / 2}}{\mu_{3.3}} \\
& H_{\mathrm{atm}, \mathrm{out}} \simeq 3.7 \mathrm{~cm} \frac{T_{\mathrm{keV}} R_{\mathrm{out}, 15}^{2}}{\mu_{3.3}} \\
& v_{\mathrm{atm}, \mathrm{th}} \simeq 9.4 \times 10^{6} \mathrm{~cm} \mathrm{~s}^{-1} \frac{T_{\mathrm{keV}}^{1 / 2}}{\mu_{3.3}^{1 / 2}},
\end{aligned}
$$

where $T_{\mathrm{keV}}$ is the atmosphere temperature in $\mathrm{keV}, R_{15}$ is the radial position in units of $15 \mathrm{~km}$, and $\mu_{3.3}$ is the mean molecular weight in units of 3.3 which represents a partially-ionized 
iron-rich atmosphere (see Sect. 3.2 in OLNII). The ring atmosphere's scale height in the $z$-direction is to be differentiated from that of the ring itself $H_{\text {ring }}$ which is of the order of a few kilometers.

The ring's atmosphere is penetrated by the magnetic field on timescales of $\sim 12$ days $T_{\mathrm{keV}, 0.1}^{5 / 2} R_{\mathrm{in}, 15 \mathrm{~km}}^{3} \mu_{\mathrm{atm} .3 .3}^{1 / 2}$. This can be derived from $\tau_{\mathrm{B}}$ (as defined Sect. 2.2) using $\delta r=H_{\mathrm{atm}}^{z}$ and $\sigma \propto 1 / c_{\mathrm{S}}$ where $c_{\mathrm{S}} \propto \sqrt{\mu / T}$ is the atmosphere's sound speed. The ring's temperature is in units of $0.1 \mathrm{keV}$ representative of its equilibrium temperature during the quiescent phase; see Eq. (16) in OLNII. Since $B^{2} / 8 \pi \gg \rho_{\mathrm{atm}} V_{\mathrm{K}}^{2}$, the atmosphere is forced to co-rotate with the field inhibiting accretion onto the star during the quiescent phase. On timescales of a hundred years, outbursts are triggered by wall accretion. These move degenerate ring material into the atmosphere (see Sect. 4 below) faster than the magnetic field lines penetrate vertically into the degenerate ring. The ring's penetration timescale is of the order of tens of millions of years because of the $(\delta r)^{2}$ dependence, so that the ring is not penetrated from above.

In our model, as we describe in more detail in Sect. 6.1 in this paper, transient AXPs do not accrete during their quiescent phase while normal AXPs do accrete continuously from the ring's outer edge, where $B^{2} / 8 \pi<\rho_{\mathrm{atm}} . V_{\mathrm{K}}^{2}$. For now we concern ourselves with transient AXPs whose quiescent phase is dominated by emission from vortex expulsion as described next.

\section{The quiescent phase in our model}

There are two critical radii in our model during the quiescent phase, the inner radius $R_{\text {in }}$, and the outer ring radius $R_{\text {out }}$. In most cases these radii will be expressed in units of $15 \mathrm{~km}$ or $25 \mathrm{~km}$ thus assigned a subscript 15 or 25 . The other 2 parameters related to the geometry of the ring are the ring's solid angle divided by $4 \pi$ at $R_{\text {in }}$ and $R_{\text {out }}$ namely, $f_{\text {in, }, \Omega}=H_{\text {ring, in }} / R_{\text {in }}$ and $f_{\text {out }, \Omega}=$ $H_{\text {ring,out }} / R_{\text {out }}$. General relativistic (GR) effects are included in the factors $f_{\mathrm{GR}, \mathrm{QS}}=\sqrt{1-R_{\mathrm{Sch} .} / R_{\mathrm{QS}}}, f_{\mathrm{GR}, \text { in }}=\sqrt{1-R_{\mathrm{Sch} .} / R_{\mathrm{in}}}$, $f_{\mathrm{GR}, \text { out }}=\sqrt{1-R_{\mathrm{Sch} .} / R_{\mathrm{out}}}$ while $f_{\mathrm{GR}, \text { ring }}=0.5\left(f_{\mathrm{GR}, \text { in }}+f_{\mathrm{GR}, \text { out }}\right)$ with $R_{\text {Sch. }}$ being the star's Schwarszhild radius. Unless otherwise specified, quantities such as luminosity, temperature, and area are local values. Values at infinity are obtained by using the relevant GR factors. Finally, the ring area which includes top, bottom and inner surfaces is $A_{\text {out }}=2 \pi\left(R_{\text {out }}^{2}-R_{\text {in }}^{2}\right)+4 \pi R_{\text {in }} H_{\text {ring, in }}=$ $2 \pi R_{\text {out }}^{2} Y_{\text {out }}$ where $Y_{\text {out }}=1-\left(R_{\text {in }} / R_{\text {out }}\right)^{2}+2\left(R_{\text {in }} / R_{\text {out }}\right)^{2} f_{\text {in }, \Omega}$.

\subsection{The 2 blackbodies in quiescence}

As discussed in OLNI and OLNII, during the quiescent phase we have 2 blackbodies, one from the emission due to magnetic reconnection following vortex expulsion (the emission occurs just outside the star's surface). The resulting luminosity is (see Sect. 5 in OLNI)

$L_{\text {vortex }} \sim 2 \times 10^{34} \mathrm{erg} \mathrm{s}^{-1} \eta_{\mathrm{X}, 0.1} \dot{P}_{-11}^{2}$,

with a corresponding temperature,

$T_{\text {vortex }} \simeq 0.2 \mathrm{keV} \eta_{\mathrm{X}, 0.1}^{1 / 4} \frac{\dot{P}_{-11}^{1 / 2}}{R_{\mathrm{QS}, 10}^{1 / 2}}$,

where the period derivative is in units of $10^{-11} \mathrm{~s} \mathrm{~s}^{-1}$, the star's radius in units of $10 \mathrm{~km}$, and $\eta_{\mathrm{X}}$ is the efficiency parameter inherent in the conversion from magnetic energy to radiation in units of 0.1 .
The second $\mathrm{BB}$ results from reprocessing by the ring of the first BB's X-ray emission, $f_{\mathrm{GR}, \mathrm{QS}}^{2} f_{\mathrm{in}, \Omega} L_{\mathrm{vortex}}=f_{\mathrm{GR}, \text { ring }}^{2} A_{\text {ring }} \sigma T^{4}$. The ring-atmosphere system's temperature during quiescence is then, since $f_{\mathrm{GR}, \mathrm{QS}} \simeq f_{\mathrm{GR} \text {,ring }}$,

$T_{\mathrm{q}} \sim 0.1 \mathrm{keV} \eta_{\mathrm{X}, 0.1}^{1 / 4} \frac{\dot{P}_{-11}^{1 / 2}}{R_{\text {out }, 25}^{3 / 8} Y_{\text {out }}^{1 / 4}}$,

where the subscript "q" stands for quiescent in contrast to the values during the bursting phase denoted by subscript " $b$ ". These two blackbodies are generic emission components to reprocess the emission from the central object, irrespective of its ultimate origin. As such, they should also be expected in normal AXPs/SGRs, where the continuous emission is dominated by the constant accretion from the ring edge rather than by vortex annihilation. Since $\dot{P} \propto t^{-2 / 3}$ in our model (Niebergal et al. 2006), both $\mathrm{BB}$ temperatures during the quiescent phase evolve in time as $t^{-1 / 3}$.

The resulting temperature ratio in our model, using a fiducial value $Y_{\text {out }} \sim 0.64$, is

$\frac{T_{\text {vortex }}}{T_{\mathrm{q}}} \sim 1.7 \frac{R_{\mathrm{out}, 25}^{3 / 8}}{R_{\mathrm{QS}, 10}^{1 / 2}}$.

Interestingly a similar correlation between the hot and cool BB for AXPs and SGRs has been observed (Nakagawa et al. 2009, see their Fig. 4), with $T_{\mathrm{BB}, \mathrm{H}} / T_{\mathrm{BB}, \mathrm{C}} \sim 2.5$. This we suggest as evidence for reprocessing in the system. The list of objects studied by Nagakawa et al. (2009) does not include transient AXPs in quiescence. These seem to be modeled by a single thermal component (e.g. Gotthelf \& Halpern 2005; Perna \& Gotthelf 2008; Bernardini et al. 2009), although because of the low fluxes, presence of the cooler component cannot be ruled out.

\section{Bursting phase in our model}

\subsection{Consequences of wall penetration and accretion}

The ring remains quiescent until magnetic penetration of the inner edge of the ring (wall). The magnetic field penetration and subsequent accretion events are very sporadic - they last for hours and occur roughly once every hundred years (see Sect. 4 in OLNII). The consequences of wall accretion are as follows:

- Change in ring's mean molecular weight: the energy released by the wall accretion is sufficient to dissociate a significant mass of iron nuclei in the ring into light nuclei (nuclei with $Z \sim 13$ ). The state of the ring depends on the Coulomb parameter which gives a solidification temperature of $T_{\mathrm{s}} \simeq 9.5 \rho_{8}^{1 / 3} \mathrm{keV}$ (see Sect. 3.2 in Ouyed \& Leahy 2009). The ring mean density (Eq. (A.8) in OLNII) is $\sim 10^{7} / t^{1 / 2} \sim 3 \times 10^{5} \mathrm{~g} \mathrm{~cm}^{-3}$, with age $t$ in yrs. This gives $T_{\mathrm{s}} \simeq 1.4 \mathrm{keV}$. However during the wall accretion event, the ring temperature rises to $\sim 4-5 \mathrm{keV}$ (see Eq. (C.2) in OLNII). This completely melts the ring, which allows light elements to rise buoyantly. Then the ring re-solidifies on a few hour timescale (Eq. (22) of OLNII). This creates a two layer system as depicted in Fig. 2. This process reduces the molecular weight of the atmosphere from $\mu_{\mathrm{q}} \sim 3.3$ to $\mu_{\mathrm{b}} \sim 2.1-2.5$ (we adopt an average $\mu_{\mathrm{b}} \sim 2.3$ ).

As estimated in Sect. 4.4 in OLNII, for a typical wall mass $m_{\mathrm{W}} \sim 10^{-10} M_{\odot}$, the number of dissociations following irradiation from wall accretion is $N_{13} \sim 10^{46} \zeta_{\mathrm{w}, 0.001} \eta_{0.1} m_{\mathrm{w},-10}$ where $\zeta_{\mathrm{w}, 0.001}$ is the dissociation efficiency in units of 0.001 , 
and $\eta_{0.1}$ is the wall accretion efficiency in units of 0.1 . The depth of the $Z \sim 13$ layer can be estimated to be $H_{13} \sim$ $10^{-3} H_{\text {ring }}$; that is, of the order of a few meters. Subsequent depletion of the $Z \sim 13$ nuclei by accretion leads to a return to an iron-rich atmosphere (i.e. $\mu_{\mathrm{q}} \sim 3.3$ ) as shown in OLNII (see also Appendix B.2 in this paper).

- The Bohm front (BF): the sudden reheating of the inner ring region triggers heat propagation outward along the ring's atmosphere. Heat propagation normal to the magnetic field can occur by either classical or Bohm diffusion.

The classical diffusion coefficient is $D_{\perp}=2 n \eta_{\perp} k_{\mathrm{B}} T c^{2} / B^{2}$ where $\eta_{\perp}=1.44 \times 10^{-} 8 Z T^{-3 / 2} \ln (\Lambda)$ is the transverse Spitzer resistivity with $\ln (\Lambda)$ the Coulomb logarithm (e.g. Eq. (5.71) in Chen 1984, for S. I. units). The Bohm diffusion coefficient is given by $c k_{\mathrm{B}} T /(16 e B)$ (see Eq. (5.111) in Chen 1984, for S. I. units). The ratio of classical to Bohm coefficients for $B=10^{13} \mathrm{G}, T=0.2 \mathrm{keV}$ and density of $10 \mathrm{~g} \mathrm{~cm}^{-3}$ is of the order of $10^{-8}$ implying that Bohm diffusion dominates over classical diffusion for magnetic field strength and temperatures involved here. Thus the heat diffuses outward according to Bohm diffusion, introducing a critical radius in our model (during the bursting phase), $R_{\mathrm{BF}}=R_{\text {in }}+\Delta r$, where $\Delta r$ is the distance that the Bohm Front (BF) has travelled from $R_{\text {in }}$ (see Appendix A for details).

- Atmosphere feeding: the boundary between the nondegenerate atmosphere and degenerate ring material, at the $\mathrm{BF}$, moves downwards into higher density layers since the $\mathrm{BF}$ heats up the interface to higher temperatures (recall that $\rho_{\mathrm{atm}} \sim T_{\mathrm{keV}}^{3 / 2}$; see Eq. (1)). Thus newly nondegenerate Keplerian ring material is unveiled between the co-rotating atmosphere and the interface (Fig. 2). We thus have two mechanisms that feed the atmosphere with new non-degenerate material. These two contributions are seen when estimating the surface density of the atmosphere $\Sigma \sim$ $\rho_{\text {atm }} H_{\text {atm }} \propto T^{5 / 2} / \mu$; the lower $\mu$ from the buoyancy and the higher $T$ from the heat front will increase the mass up by a factor of $\sim 10$.

- Atmosphere ejection and accretion: the huge shear between the co-rotating, magnetized atmosphere and the underlying non-degenerate keplerian atmosphere leads to Kelvin-Helmholtz instability that helps load the keplerian material onto the magnetic field lines attached to the heat front $^{2}$. With simple angular momentum arguments one can show that any keplerian particle attached to a co-rotating field line will slide along the field line moving radially outward. The magnetic field acts as ramp for the plasma particles to get rid of their excess angular momentum. This is the slingshot effect in magneto-hydrodynamic (MHD) jets (discussed in Appendix B). In the quiescent state there is a huge shear between the corotating field lines (containing the nondegenerate atmosphere) and the Keplerian degenerate ring material. However since the field lines do not penetrate the degenerate ring no MHD ejection (and thus no accretion) is feasible.

In summary, the K-H instability would load mass onto the field line attached to the Bohm front and get ejected centrifugally along the field lines as explained in Appendix B (see also below). The wind is then channeled onto the star

\footnotetext{
2 During quiescence the shear is between the solid degenerate Keplerian ring and the co-rotating atmosphere thus not prone to the instability. In contrast, during burst the shear is between one fluid (the new Keplerian atmosphere) and another fluid (the pre-existing co-rotating atmosphere).
}

with an accretion rate, $\dot{m}_{\text {acc., }}$ given by Eq. (B.2) creating a hopt spot (HS) on the surface of the star.

\subsection{The 2 blackbodies during burst}

Only magnetic field lines that are in the path of the heat front get loaded and accrete onto the star. The main consequence, as we show in details here, is that the HS on the star moves closer to the pole thus decreasing in area.

The temperature of the inner ring is obtained by equating heating from BF induced accretion onto the quark star with blackbody cooling by the inner ring surface $\left(R<R_{\mathrm{BF}}\right)$; $f_{\mathrm{GR}, \mathrm{QS}}^{2} f_{\mathrm{BF}, \Omega} L_{\mathrm{acc}}=f_{\mathrm{GR}, \text { ring }}^{2} A_{\mathrm{BF}} \sigma T_{\mathrm{b}}^{4}$ where $L_{\mathrm{acc}}=\eta \dot{m}_{\mathrm{acc} .} c^{2}$ is the accretion luminosity, $\eta$ the accretion efficiency, $f_{\mathrm{BF}, \Omega}=$ $H_{\text {ring,BF }} / R_{\mathrm{BF}}$, and the area created by the Bohm front is $A_{\mathrm{BF}}=$ $2 \pi\left(R_{\mathrm{BF}}^{2}-R_{\text {in }}^{2}\right)+4 \pi R_{\text {in }} H_{\text {ring, in }}=2 \pi R_{\mathrm{BF}}^{2} Y_{\mathrm{BF}}$. Here $Y_{\mathrm{BF}}=1-$ $\left(R_{\mathrm{in}} / R_{\mathrm{BF}}\right)^{2}+2\left(R_{\mathrm{in}} / R_{\mathrm{BF}}\right)^{2} f_{\mathrm{in}, \Omega}$. The resulting temperature is,

$T_{\mathrm{b}} \simeq 0.21 \mathrm{keV} \eta_{0.1} \zeta_{\mathrm{sp}, 0.01} \frac{R_{\mathrm{BF}, 15}}{\mu^{3 / 2} Y_{\mathrm{BF}}}$

Here, $\zeta_{\mathrm{sp}, 0.01}$ in units of 0.01 , is a factor related to mass-loading at the slow MHD point as determined in Appendix B. The approximation above is valid until late times when accretion ceases, so that $T_{\mathrm{b}}$ is no longer given by the accretion-cooling balance but rather settles to the quiescent temperature $T_{\mathrm{q}}$. The outer ring's temperature is given by the quiescent temperature, Eq. (4), until the heat front reaches it.

The accretion rate is then obtained by combining Eq. (B.2) with equation above to get

$\dot{m}_{\text {acc. }} \sim 1.6 \times 10^{15} \mathrm{~g} \mathrm{~s}^{-1} \eta_{0.1}^{3} \zeta_{\mathrm{sp}, 0.01}^{4} \frac{R_{\mathrm{BF}, 15}^{11 / 2} f_{\mathrm{K}}\left(T_{\mathrm{b}}\right)}{\mu^{6} Y_{\mathrm{BF}}^{3}}$.

The function $f_{\mathrm{K}}\left(T_{\mathrm{b}}\right)$ is described in Appendix B and acts as a "valve" that shuts-off accretion after the heating front has passed. There is a fixed amount of Keplerian material at any radius that can be fed into an MHD wind and thus accreted onto the star. This is because of re-enforced co-rotation once the magnetic field penetrates the non-degenerate Keplerian layer effectively shutting off accretion (see Fig. 2).

The natural connection between the BF outward expansion and the resulting HS on the star is illustrated in Fig. 2. The corresponding HS luminosity is

$L_{\text {acc. }} \sim 1.4 \times 10^{35} \mathrm{erg} \mathrm{s}^{-1} \eta_{0.1}^{4} \zeta_{\mathrm{sp}, 0.01}^{4} \frac{R_{\mathrm{BF}, 15}^{11 / 2} f_{\mathrm{K}}\left(T_{\mathrm{b}}\right)}{\mu^{6} Y_{\mathrm{BF}}^{3}}$,

with a HS temperature

$T_{\mathrm{HS}} \sim 0.58 \mathrm{keV} \eta_{0.1} \zeta_{\mathrm{sp}, 0.01} \frac{R_{\mathrm{BF}, 15}^{11 / 8} f_{\mathrm{K}}\left(T_{\mathrm{b}}\right)^{1 / 4}}{\mu^{3 / 2} Y_{\mathrm{BF}}^{3 / 4} A_{\mathrm{HS}, 120}^{1 / 4}}$,

where the HS area, $A_{\mathrm{HS}}$ is in units of $120 \mathrm{~km}^{2}$, is derived in the Appendix (see Eq. (C.1)).

In summary, during the burst phase, the ring-atmosphere system consists of $2 \mathrm{BBs}$; the inner warmer part increasing in area at the Bohm diffusion rate and the outer cooler part decreasing in area. The hot BB in our model is provided by the HS which decreases in area as the BF propagates outwards. 

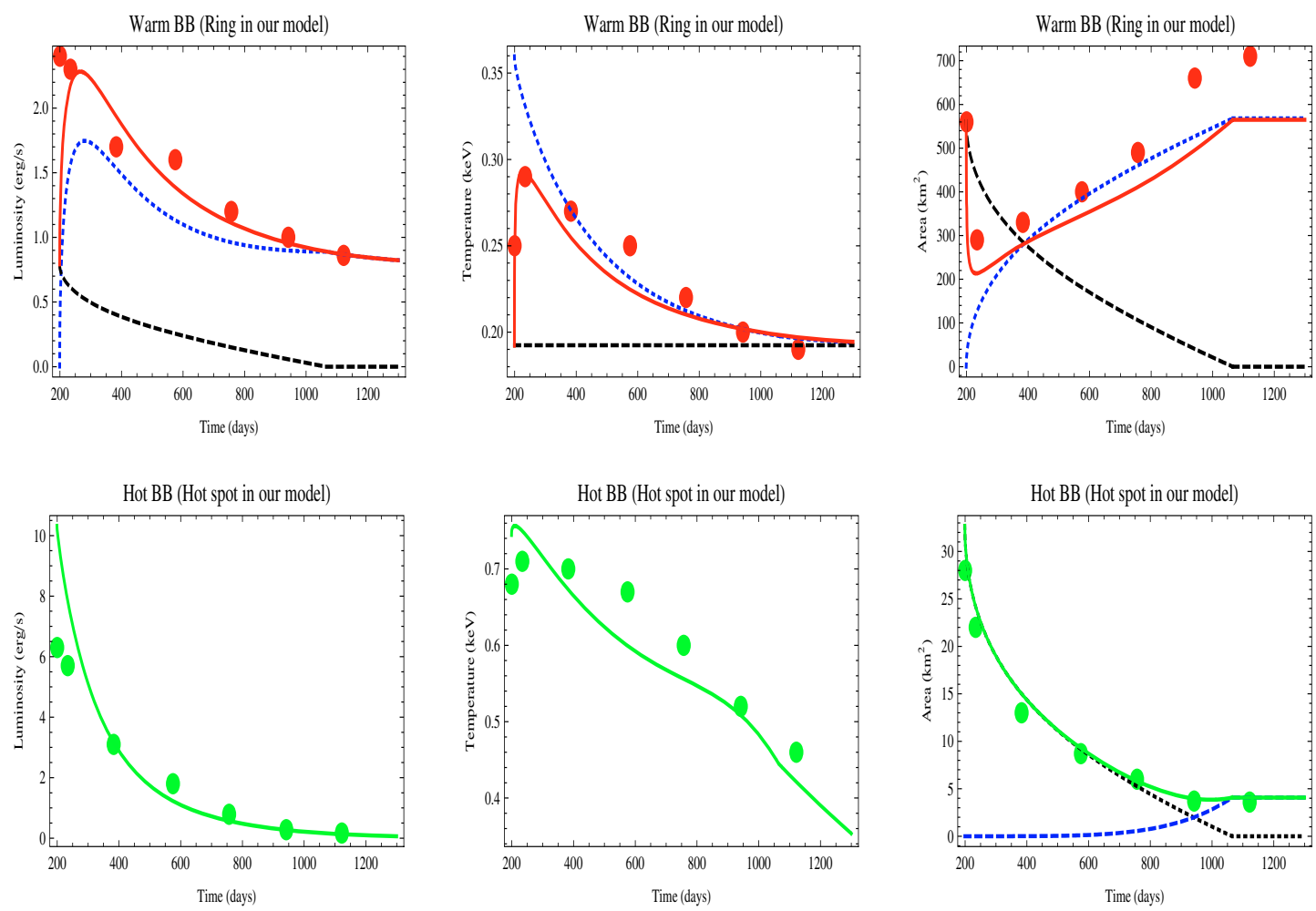

Fig. 3. Upper panels: model luminosity $\left(L / 10^{34}\right)$, temperature and area (curves) from one side of the ring as observed at infinity compared to observations (dots) of the warm component of XTE J1810-197. The dotted line is the contribution inward of the BF, the dashed is from outward of the BF, and the solid is the combined contribution (see text). Lower panels: model luminosity $\left(L / 10^{34}\right)$, temperature and area (curves) from one pole as observed at infinity compared to observations (dots) of the hot component of XTE J1810-197. In the right-most panel the dotted line traces the shrinkage of the HS as the BF moves outward, the dashed line shows the late contribution from accretion induced by heating of the back side of the ring, and the solid is the combined contribution.

\subsection{Temperature ratio during burst}

The temperature ratio in the bursting phase case is then:

$\frac{T_{\mathrm{HS}}}{T_{\mathrm{b}}}=\left(\frac{A_{\mathrm{BF}}}{f_{\mathrm{BF}, \Omega} A_{\mathrm{HS}}}\right)^{1 / 4} \sim 2.8\left(\frac{R_{\mathrm{out}, 25}}{R_{\mathrm{QS}, 10}}\right)^{1 / 2}$,

where we approximated $A_{\mathrm{BF}} \sim A_{\text {ring }} / 2, f_{\mathrm{BF}, \Omega} \sim f_{\text {out }, \Omega}$, and $A_{\mathrm{HS}} \sim 0.1 A_{\mathrm{QS}}$ to get the numerical value. Our model predicts the temperature ratio during burst for a given object to be higher by a factor of $\sim 1.5$ than during quiescence. We expect a scatter in comparing different objects mainly caused by variations in $R_{\text {out }}$. This is consistent with Fig. 4 of Nakagawa et al. (2009).

\subsection{Application to XTE J1810-197}

The upper panels in Fig. 3 compares the time evolution of the ring's luminosity, temperature and area in our model to the observed warm BB in XTE J1810-197. In our model the luminosity from the inner part of the ring is $L_{\mathrm{BF}}=A_{\mathrm{BF}} \sigma T_{\mathrm{b}}^{4}$ while the outer part gives $L_{\text {out }}=A_{\text {out }} \sigma T_{\mathrm{q}}^{4}$ with $A_{\text {out }}=2 \pi\left(R_{\text {out }}^{2}-R_{\mathrm{BF}}^{2}\right)$. The total luminosity from the ring during burst is $L_{\text {ring, } \mathrm{b}}=L_{\mathrm{BF}}+L_{\text {out }}$ while the corresponding effective area and temperature were derived by weighting over luminosity, $A_{\text {ring,eff. }}=\left(A_{\mathrm{BF}} L_{\mathrm{BF}}+\right.$ $\left.A_{\text {out }} L_{\text {out }}\right) / L_{\text {ring, } \mathrm{b}}$ and $T_{\text {ring,eff. }}=\left(T_{\mathrm{b}} L_{\mathrm{BF}}+T_{\mathrm{q}} L_{\text {out }}\right) / L_{\text {ring, } \mathrm{b}}$.

The lower panels in Fig. 3 compare the time evolution of the HS's luminosity, temperature and area in our model to the observed hot BB in XTE J1810-197. In this case, only one component comes into play, the accretion luminosity $L_{\text {acc. }}$. However, as the BF gets closer to $R_{\text {out }}$ the back side of the ring is heated resulting in additional accretion by the same mechanism (i.e. eating into Keplerian material in the radial direction this time) and additional area on the polar cap defined by the field lines connecting the back side of the ring to the star (see Fig. 2). The resulting area is $A_{\mathrm{HS}}+A_{\text {edge }}$ and temperature $\left(L_{\mathrm{acc}} /\left(\sigma\left(A_{\mathrm{HS}}+\right.\right.\right.$ $\left.\left.\left.A_{\text {edge }}\right)\right)\right)^{1 / 4}$.

The fits to XTE J1810-197 data were obtained for the following set of parameters:

$R_{\text {in }}=23.5 \mathrm{~km}$

$R_{\text {out }}=26.5 \mathrm{~km}$,

and by slightly adjusting the mass-load at the slow MHD point so that $\zeta_{\mathrm{sp}}=0.02$ (see discussion following Eq. (B.1)). The other parameters were kept to their fiducial values including the star's parameters $R_{\mathrm{QS}}=10 \mathrm{~km}, M_{\mathrm{QS}}=1.4 M_{\odot}$. The star's magnetic field is given by $B_{\mathrm{QS}}=\sqrt{3 \kappa P \dot{P}} \sim 3.3 \times 10^{14} \mathrm{G}($ with $\kappa=8.8 \times$ $10^{38} \mathrm{G}^{2} \mathrm{~s}^{-1}$ as given in Eq. (10) in OLNIII).

Assuming that XTE J1810-197 has experienced a few bursting events, using Eq. (A.7) in OLNII, the ring would have spread to no more than $(\Delta r)_{\mathrm{t}} \sim 8 \mathrm{~km}$ if the system's temperature during quiescence remained on average $\sim 0.1 \mathrm{keV}$. This is consistent with $\left(R_{\text {out }}-R_{\text {in }}\right)$ found from fits to the XTE J1810-197 data thus providing a self-consistency check on our model. This also confirms our overall findings in previous work (OLNI, OLNII and OLNIII) that the ring should be a few kilometers in width after a few hundred years.

\subsection{Application to 1E 1547.0-5408}

In its bursting phase, this source was fitted with a $T \sim 0.5$ $\mathrm{keV}$ hot $\mathrm{BB}$ with $\left.L_{\mathrm{BB}} \sim 1.3 \times 10^{35}(d / 9 \mathrm{kpc})^{2}\right) \mathrm{erg} \mathrm{s}^{-1}$ and a 
corresponding area decreasing from $180 \mathrm{~km}^{2}$ in June 2007 to $96 \mathrm{~km}^{2}$ in August 2007 (see Halpern et al. 2008). During the quiescent phase, $\left.L_{\mathrm{BB}} \sim 10^{34}(d / 9 \mathrm{kpc})^{2}\right) \mathrm{erg} \mathrm{s}^{-1}, T_{\mathrm{BB}} \sim 0.4 \mathrm{keV}$ and $A_{\mathrm{BB}} \sim 36 \mathrm{~km}^{2}$ (see Table 1 in Halpern et al. 2008).

This source has not been as well sampled as was XTE J1810-197. Nevertheless, in our model, high BB temperatures during the quiescent phase are suggestive of a more compact ring which is closer to the star (see Eq. (4)). In our model, such a small and compact ring could have been a consequence of a smaller amount of crust material ejected during the QN (see Eq. (2) in OLNII). For example, $R_{\text {out }}=15 \mathrm{~km}$ and $R_{\text {in }}=13 \mathrm{~km}$, which implies $Y_{\text {out }} \sim Y_{\mathrm{BF}} \sim 0.117$, inserted in Eq. (4) gives $T_{\mathrm{q}} \sim 0.35 \mathrm{keV}$; we take $\dot{P}_{-11} \sim 2 \mathrm{~s} \mathrm{~s}^{-1}$ for this source. The ring's burst epoch temperature is then $\sim 0.48 \mathrm{keV}$ with a corresponding peak luminosity from Eq. (8) of $L_{\text {acc. }} \sim 4.8 \times 10^{35} \mathrm{erg} \mathrm{s}^{-1}$ for $\mu_{\mathrm{b}}=2.4$. This is close to the $\left.\sim 1.3 \times 10^{35}(d / 9 \mathrm{kpc})^{2}\right) \mathrm{erg} \mathrm{s}^{-1}$ measured in June-July 2007 (the peak of the outburst was not observed and could have been higher than this). The initial area of the HS is given by Eq. (C.1) and is estimated to be $\sim 80 \mathrm{~km}^{2}$ using $R_{\text {in }}=13 \mathrm{~km}$ and $R_{\text {out }}=15 \mathrm{~km}$. Finally, in the case of $1 \mathrm{E}$ 1547.0-5408 there seems to be hints of an aligned rotator from its small X-ray pulsed fraction and its relatively broad radio pulse. In our model, the QN compact remnant (the QS) is born as an aligned rotator due to the vortex confinement of magnetic field (see Fig. 1).

\section{The radio emission in our model}

In this section we develop a scenario for radio emission. As discussed in Sect. 1.2, XTE J1810-197 and 1E 1547.0-5408 show unique characteristics in radio (including delay after X-ray outburst, a flat spectrum, and unusual spin-down behavior). In chronological order the sequence of events that leads to radio emission in our model is as follows:

- magnetic bubbles are generated at the ring during the X-ray outburst (see Sect. 5.1);

- the propagation time of these bubbles from the site of production (the ring) to the 1.c. defines the delay between radio and X-ray outbursts (see Sect. 5.2);

- the unusual spin-down behavior is induced by torques on the magnetosphere from the co-rotating bubbles, during their outward propagation (see Sect. 5.5);

- relativistic collisionless reconnection at the 1.c. destroys the bubbles;

- magnetic energy released by the bubbles is eventually radiated by particles accelerated by reconnection at the 1.c., mostly at radio frequencies (Sect. 5.3). We suggest that the flat radio spectrum is naturally associated to this mechanism (see Sect. 5.4 below).

\subsection{Magnetic reconnection and bubble generation}

After penetration, the poloidal magnetic field $\left(B_{\mathrm{p}}\right)$ lines inside the wall (inner ring) are dragged by the Keplerian shear generating a toroidal magnetic field, $B_{\phi}$. Continuous reconnection events during the winding of the field lines lead to X-point generation and emergence of closed magnetic bubbles; more concisely these magnetically confined plasma bubbles (plasmoids) result from the Keplerian shear.

Generation of bubbles is a common feature of threaded disks as seen in many simulations (e.g. Romanova et al. 1998; Yelenina et al. 2006). These simulations show the magnetic bubbles to acquire enough speed to escape gravity and expand freely outward. Magnetic loops and bubbles are also common in the sun (referred to as plasmoids) and are also found to gain enough energy from the reconnection events to escape the system and expand freely (e.g. Wagner 1984; Tamano 1991).

One key difference between these cases and our model is the fact that in our model, during quiescence, the degenerate ring is not threaded by the magnetic field. The bubbles form only during the bursting phase once the magnetic field has penetrated the inner ring and is sheared. The $B$ field attached to the broken inner pieces of the wall is wound up by the keplerian angular velocity $(\sim 6000 \mathrm{rad} / \mathrm{s})$ of the piece vs. the corotation angular velocity $(\sim 1 \mathrm{rad} / \mathrm{s})$ of the footpoint of the fieldline on the quark star. This rapid field line winding results in reconnection and magnetic loop (bubble) formation. The $B$ field penetration (and subsequent wall accretion with concurrent bubble generation) events are very sporadic and unique to our model - they last for about an hour and occur once every century (see Sect. 4 in OLNII).

A rough estimate of the number of bubbles that can be generated is

$N_{\text {Bub. }} \sim \frac{V_{\mathrm{w}}}{V_{\text {Bub. }}} \sim 8.3 \times 10^{6} R_{\mathrm{in}, 15}^{3 / 2}$,

where the total reconnection volume is $V_{\mathrm{w}} \sim 4 \pi R_{\text {in }} H_{\text {ring,in }} \delta r_{\mathrm{w}}$ and $V_{\mathrm{Bub}} \sim(4 \pi / 3) \delta r_{\mathrm{w}}^{3}$. The mass of a given bubble is thus $m_{\text {Bub. }} \sim(4 \pi / 3) \delta r_{\text {w }}^{3} \rho_{\text {atm.,w }}$ where the plasma confined by the bubbles has a density at birth given by the atmosphere density $\rho_{\text {atm.,w }}$. The magnetic energy stored in each bubble is then $E_{\text {Bub. }} \sim(4 \pi / 3) \delta r_{\mathrm{w}}^{3}\left(B_{\phi}^{2} / 8 \pi\right)$ with $B_{\phi} \sim B_{\mathrm{p}, \text { in }}$ with $B_{\mathrm{p}, \text { in }}=$ $B_{\mathrm{QS}}\left(R_{\mathrm{QS}} / R_{\mathrm{in}}\right)^{3}$.

\subsection{Delay between the $X$-ray and radio emission}

Simulations of bubbles in threaded disks show that magnetic loops and bubbles propagate outward because of the energy they gain from reconnection and because of the dipolar $B$-field gradient from the central star, similar to the way solar field disturbances propagate outward above the solar photosphere. These bubbles propagate at the sound speed of non-dissipative magnetic field disturbances, i.e. at the Alfvén speed. Illustrated in Fig. 4 in this paper, is a rudimentary representation of what is seen in these simulations; an outward migration process through the dipole field involving reconnection. This is a simple and only qualitative model for bubble propagation, which is a propagating geometric disturbance in the magnetic field/plasma. Bubble propagation results in different field lines and different plasma making up the bubble as it moves. As seen from Fig. 4, a magnetic field line in front of the bubble is only temporarily part of the bubble, and leaves the bubble after the bubble has propagated past it. The reconnection on the front side of the bubble results in surface pressure similar to the static $B^{2} / 8 \pi$, so the dynamics of reconnection during bubble migration are important to how the bubble evolves (both its speed and change in volume) as it migrates.

The Alfvén velocity depends on the magnetic field geometry inside the light cylinder (which we take to be nearly dipolar) and on the ambient density which we take to scale as $\propto\left(R_{\text {in }} / r\right)^{\alpha}$. If there is a steady wind inside the magnetosphere, then if the wind is spherically symmetric the density we expect would decline as $r^{-2}$, or if it is equatorially confined the density would decline as $r^{-1}$; that is $1 \leq \alpha \leq 2$. Although the exact distribution is not at all known (e.g. Michel 1969; Spitkovsky 2008; see more discussion on this aspect of our model in 6.5) we will adopt $\alpha=1$ 


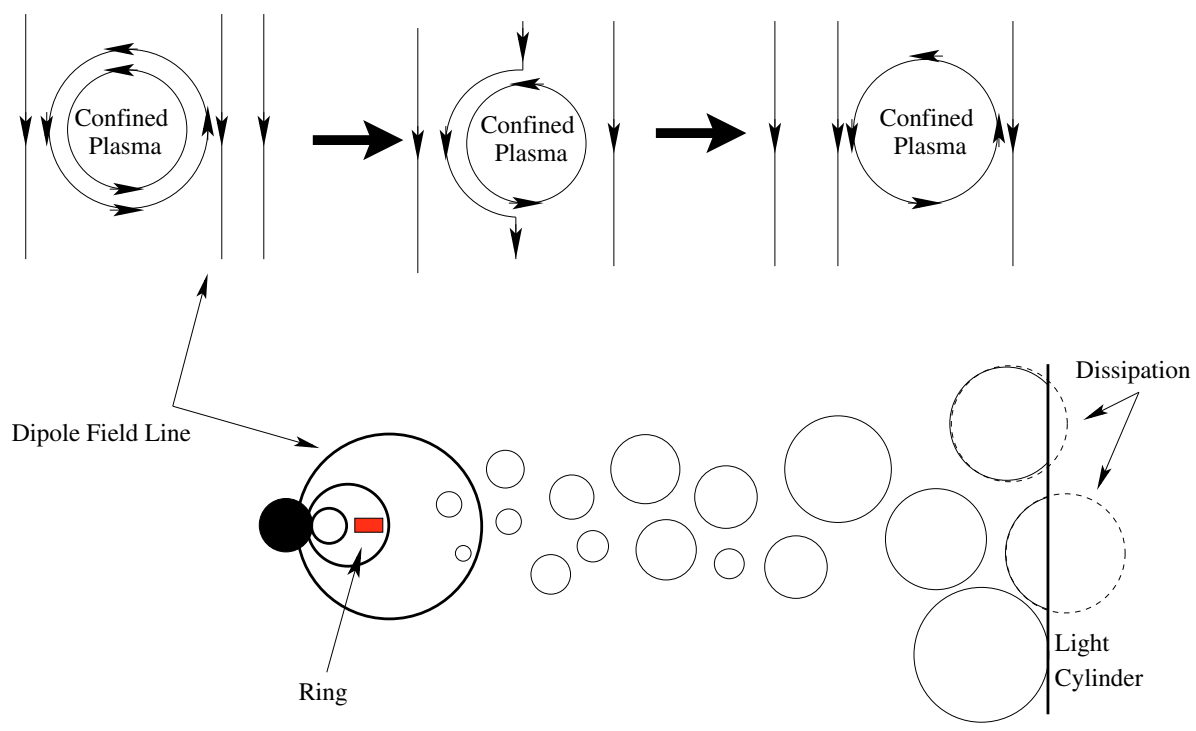

Fig. 4. Keplerian shear during wall accretion leads to X-point reconnection which in turn causes the generation of closed magnetic loops, or "bubbles" (see Sect. 5.1 in text). In our model, these magnetic bubbles are responsible for the radio emission seen in some AXPs. The top panel illustrates the outward migration of a bubble through the surrounding magnetic field. The bubble's magnetic polarity in front as it moves outward is opposite to that of the surrounding dipole. This leads to reconnection and the motion of the bubble towards the light cylinder. The lower diagram shows the overall pattern of bubble migration (i.e. magnetic buoyancy) and expansion that leads to pile up near the light cylinder. The bubbles dissipate as they cross the light cylinder leading to the radio emission (see text for details). as we expect the bubbles to remain along the equator since they are Keplerian at birth.

The bubbles move outwards at a rate given by $\mathrm{d} r / \mathrm{d} t=v_{\mathrm{A}}$ where $v_{\mathrm{A}}=c\left(R_{0} / r\right)^{3-\alpha / 2}$ so that $R_{0} \sim$ $45 \mathrm{~km}\left(B_{\mathrm{QS}, 14} / T_{\mathrm{atm}, \mathrm{keV}}^{5 / 4}\right)^{1 /(3-\alpha / 2)}$ is the radius beyond which the Alfvén speed becomes sub-relativistic; the star's magnetic field $B_{\mathrm{QS}, 14}$ is in units of $10^{14} \mathrm{G}$.

The time it takes the magnetic bubbles to reach the light cylinder (lc) at $R_{\mathrm{lc}}=c / \Omega$ (at which point they start dissipating) is found from $\int_{R_{0}}^{R_{\mathrm{lc}}} \mathrm{d} r / v_{\mathrm{A}}$,

$t_{\text {prop. }} \sim \frac{1}{4-\frac{\alpha}{2}}\left(\frac{R_{0}}{c}\right)\left(\frac{R_{\mathrm{lc}}}{R_{0}}\right)^{4-\frac{\alpha}{2}} \propto \frac{P^{\frac{7}{2}-\frac{\alpha}{2}}}{\dot{P}^{1 / 2}}$,

where we made use of the definition of $R_{0}$ and $B_{\mathrm{QS}} \propto \sqrt{P \dot{P}}$ in our model. For example for $\alpha=1$, we get

$t_{\text {prop. }} \sim 4.1$ yrs $\frac{P_{5}^{3} T_{\mathrm{atm} . \mathrm{keV}}^{5 / 4}}{\dot{P}_{-11}^{1 / 2}}$,

where the period is given in units of $5 \mathrm{~s}$; recall that $B_{\mathrm{QS}}=$ $3.6 \times 10^{14} \mathrm{G} \sqrt{P_{5} \dot{P}_{-11}}$. As they slow down while flowing towards the lc the bubbles will pile-up. This implies a delay between the X-ray outburst following wall accretion and the peak of radio emission in our model. Thus, following the peak of the X-ray outburst for XTE J1810-197 (with $P \simeq 5.54 \mathrm{~s}$ ) and 1E 1547.0-5408 (with $P \simeq 2$ s), we expect a corresponding radio peak emission to occur with a delay of $\sim 320$ days and $\sim 15$ days respectively (assuming $T_{\text {atm. }} \sim 0.3 \mathrm{keV}$ and $\dot{P}_{-11} \sim 2$ ).

\subsection{Duration and luminosity}

The radio duration has two components in our model: (i) the radiative lifetime of the electrons, $t_{\text {cool }}$; (ii) the time difference between when the first bubble arrives and the last bubble arrives at the 1.c., $t_{\text {radio }}=\Delta t_{\text {prop. }}$. As we show at the end of this section, propagation delays between bubbles is the dominant component. Below we focus on case (ii).

Since all bubbles are produced within a few hours (during the X-ray burst) and at the same location, the radio duration is determined by variations in propagations times of the bubbles to the 1.c. The magnetic reconnection events lead to variations in $B$ which translate to fluctuations in $v_{\mathrm{A}}$ and thus propagation time (consequently arrival time at the 1.c.) resulting in $t_{\text {radio }}=$ $\Delta t_{\text {prop. }}=\left(t_{\text {prop.,max }}-t_{\text {prop.,min }}\right)=t_{\text {prop }} \times(\Delta B / B)$ where $(\Delta B / B)=$ $\left(B_{\max }-B_{\min }\right) / B$. Or,

$t_{\text {radio }} \simeq t_{\text {prop. }} \times \frac{\Delta B}{B}$.

For a homologous expansion during the outward propagation, a given bubble increases in size as $\delta r_{\mathrm{Bub}} \sim \delta r_{\mathrm{w}}\left(r / R_{\text {in }}\right)$. Combined with flux conservation, $B_{\text {Bub. }} \propto \delta r_{\text {Bub. }}^{-2}$ and $V_{\text {Bub. }} \propto \delta r_{\text {Bub. }}^{3}$, it yields $E_{\mathrm{Bub}} .=V_{\mathrm{Bub} .} B_{\mathrm{Bub} .}^{2} /(8 \pi) \propto r^{-1}$. The radio luminosity is then given as, for $\alpha=1$,

$$
\begin{aligned}
L_{\text {radio }} \simeq & \eta_{\mathrm{R}} \frac{E_{\text {mag.,1.c. }}^{\text {Bub. }}}{t_{\text {radio }}} \\
\sim & 4.4 \times 10^{29} \mathrm{erg} \mathrm{s}^{-1} \times \eta_{\mathrm{R}} \times \frac{B}{\Delta B} \\
& \times \frac{\dot{P}_{-11}^{3 / 2} R_{\mathrm{QS}, 10}^{6} \rho_{\text {ring, } 9}^{1 / 6}}{P_{5}^{3} R_{\text {in }, 15} T_{\mathrm{atm} . \mathrm{keV}}^{5 / 4}},
\end{aligned}
$$

where $E_{\text {mag.,l.c. }}^{\text {Bub. }}=E_{\text {mag.,in }}^{\text {Bub. }} \times\left(R_{\text {in }} / R_{\text {l.c. }}\right) \sim\left(\frac{B_{\text {p.in }}^{2}}{8 \pi} V_{\mathrm{w}}\right) \times\left(R_{\text {in }} / R_{\text {l.c. }}\right)$ is the total magnetic energy stored in the bubbles by the time they reach the light cylinder. The ratio $\left(R_{\text {in }} / R_{\text {l.c. }}\right)$ is the dilution factor induced by the homologous expansion of the bubbles as they propagate from $R_{\text {in }}$ to $R_{\text {l.c. }}$; recall that $B_{\text {in }}=B_{\mathrm{QS}}\left(R_{\mathrm{QS}} / R_{\text {in }}\right)^{3}$. In the equation above, $\eta_{\mathrm{R}}$ is the efficiency of conversion of magnetic energy to radio emission via reconnection (e.g. Ouyed et al. 2006) at the 1.c.

For XTE J1810-197, Camilo et al. (2007c) report radio emission lasting at least 272 days with initial fading from $5 \mathrm{mJy}$ to $2 \mathrm{mJy}$ in the first few weeks (see their Fig. 2). In our model, $t_{\text {radio }} \sim 272$ days gives $\Delta B / B \sim 0.85$. Then using $R_{\text {in }} \simeq 23.5 \mathrm{~km}$ (from the X-ray fits in Sect. 4.4), $L_{\text {radio }} \simeq$ $\eta_{\mathrm{R}} \times 3 \times 10^{30} \mathrm{erg} \mathrm{s}^{-1}$. This matches the observed radio luminosity of $\sim 2 \times 10^{30} \mathrm{erg} \mathrm{s}^{-1}$ for $\eta_{\mathrm{R}} \sim 0.7$ which is suggestive of a high dissipation/reconnection efficiency of the bubbles at the l.c.

For 1E 1547-5408, radio emission was observed (Camilo et al. 2008) from June through August 2007 ( $~ 90$ days). It was not detected Jan. 22, 2009 within $18 \mathrm{~h}$ of the first reports of renewed X-ray (SGR) bursting activity from it (Camilo et al. 2009). In our model, this is suggestive of $\Delta B / B \sim 6.0$. Then 
using $R_{\text {in }} \simeq 13 \mathrm{~km}$ (from the X-ray fits in Sect. 4.5), $L_{\text {radio }} \simeq$ $\eta_{\mathrm{R}} \times 2 \times 10^{32} \mathrm{erg} \mathrm{s}^{-1}$. This matches the observed radio luminosity of $\sim 2 \times 10^{30} \mathrm{erg} \mathrm{s}^{-1}$ for $\eta_{\mathrm{R}} \sim 1 \%$.

The fits above indicate an order of magnitude difference in efficiency, $\eta_{\mathrm{R}}$, between the two sources. However, a smaller $\alpha$ (i.e. a slower density decrease inside the l.c.) for 1E 1547-5408 would lead to a longer propagation time and lower radio luminosity, implying a higher reconnection efficiency. Alternatively, a more compact quark star for 1E 1547-5408 would also imply a higher reconnection efficiency.

As a given bubble reaches the 1.c. it is sheared and spreadout around the equator of the 1.c. The resulting bubble's magnetic field is given by $B_{1 . c .}^{\text {Bub. }}\left(\delta r_{1 . c .}^{\text {Bub. }}\right)^{2}=B_{\text {sh. }}^{\text {Bub. }}\left(\delta r_{1 . c .}^{\text {Bub. }}\right) \times 2 \pi R_{\text {l.c. }}$. This yields

$B_{\text {sh. }}^{\text {Bub. }} \sim 60 \mathrm{G} \frac{\dot{P}_{-11}^{1 / 2} R_{\mathrm{QS}, 10}^{3}}{P_{5}^{3 / 2} R_{\mathrm{in}, 15}^{1 / 2}}$.

After shearing, reconnection occurs accelerating electrons (see Sect. 5.4). The electrons emit synchrotron radiation with a critical frequency of $v_{\mathrm{c}}=0.42 \mathrm{GHz} \gamma_{10}^{2} B_{\text {sh.,G }}^{\text {Bub. }}$ where $\gamma_{10}$ is the electron Lorentz factor in units of 10 following reconnection and the sheared bubble magnetic field is in units of Gauss. We get

$v_{\mathrm{c}} \sim 25 \mathrm{GHz} \gamma_{10}^{2} \frac{\dot{P}_{-11}^{1 / 2} R_{\mathrm{QS}, 10}^{3}}{P_{5}^{3 / 2} R_{\mathrm{in}, 15}^{1 / 2}}$.

For XTE J1810-159 the above gives $v_{\mathrm{c}} \sim 24 \mathrm{GHz}$ while it is $v_{\mathrm{c}} \sim 150 \mathrm{GHz}$ for $1 \mathrm{E} 1547-5408$. Observations at higher frequencies than currently available could constrain our model.

The above calculations neglect the radiative lifetime of the electrons. However in cases where the bubbles reach the 1.c. simultaneously (i.e. with negligibly short time delays), the radio duration will be dominated by the radiative cooling lifetime of the electrons, $t_{\text {cool }} \sim 0.25$ day $/\left(\gamma_{10} B_{\text {sh.,60 }}^{2}\right)$ (Lang 1999). For our estimated values of $\gamma$ and $B_{\text {sh. }}$, the propagation delay is much longer than the electron radiative lifetime. E.g. for 1E 1547-5408, the radiative lifetime only becomes important, with $\gamma=10$, for $B_{\text {sh. }}<3 \mathrm{G}$, which would also result in the critical synchrotron $v_{\mathrm{c}}$ below the observed radio frequencies.

\subsection{The flat spectrum}

As they cross the lc, the bubbles will dissipate by braking up into smaller bubbles while driving complex, non-linear reconnection events. Particle in Cell simulations of X-point reconnection events in a pair plasma have shown that highly variable radio emission, with an extremely flat spectrum $(s \sim-0.17)$ is a natural outcome with pairs accelerated to Lorentz factors of up to $\sim 70$ (e.g. Fig. 2 in Jaroschek et al. 2004). The flat spectrum is observed up to the cutoff frequency at $\sim 100 \mathrm{GHz}$ (e.g. Fig. 4 in Jaroschek et al. 2004). These simulations show that the highly dynamic non-linear evolution of thin current sheets serve as the fundamental plasma scenario to obtain a flat synchrotron power spectra in pair-dominated environments. The emitted synchrotron emission is highly linearly polarized, with spectral polarization varying from $>50 \%$ around $1 \mathrm{GHz}$ and up to $80 \%$ or higher at higher frequencies.

If pair generation regions exist in the vicinity of the lc then, when combined with magnetic reconnection events from bubble dissipation, it would offer a natural explanation for the observed flat spectrum. This could also help account for the prevalence of emission at a particular rotation phase (see Fig. 2 in Camilo et al. 2006) which implies that the co-rotating bubbles must preferentially dissipate at a particular co-rotating longitude. It might also be the case that there exist a small non-uniformity at the light cylinder, caused by feedback from pairs produced by bubble dissipation mechanism itself.

\subsection{Torques during radio emission}

As each bubble expands toward the light cylinder it exerts a torque $\tau_{\mathrm{Bub} .}=\Omega \times \mathrm{d} I_{\mathrm{Bub} .} / \mathrm{d} t=\Omega \times\left(2 m_{\mathrm{Bub} .} r v_{\mathrm{A}}+\dot{m}_{\mathrm{Bub} .} r^{2}\right)$ where $I_{\mathrm{Bub}}=m_{\mathrm{Bub}} r^{2}$ is the moment of inertia of a bubble at a radius $r$ from the star; here $\dot{m}_{\mathrm{Bub}}$ is the rate of change of the bubble mass. For $\dot{m}_{\mathrm{Bub}}=0$, the total torque exerted, $\tau=N_{\mathrm{Bub} .} \tau_{\mathrm{Bub}}$, is then

$\tau \sim 8.8 \times 10^{32}$ dyn $\mathrm{cm} \frac{R_{\mathrm{in}, 15}^{6}}{P_{5}} t_{\text {days }}^{-3 / 7}$.

The corresponding frequency derivative, $\dot{v}=-\tau /\left(2 \pi I_{\mathrm{QS}}\right)$ with $I_{\mathrm{QS}} \sim 10^{45} \mathrm{~g} \mathrm{~cm}^{2}$ for the star, is

$\dot{v} \sim-1.4 \times 10^{-13} \mathrm{~s}^{-2} \frac{R_{\mathrm{in}, 15}^{6}}{P_{5} I_{\mathrm{QS}, 45}} t_{\text {days }}^{-3 / 7}$.

This gives $\dot{v} \sim-1.9 \times 10^{-12} \mathrm{~s}^{-2}$ and $\dot{v} \sim-10^{-13} \mathrm{~s}^{-2}$ for XTE J1810-197 and 1E 1547.0-5408, respectively, which is of the right order of magnitude compared to what is observed.

In our model, the torque decreases in time (as the X-ray decreases) in accordance with what has been observed in the case of XTE J1810-197 (Camilo et al. 2007c). However, for $1 \mathrm{E} 1547.0-5408$, the torque increased in time as the X-ray flux decreased. To explain the case of 1E 1547.0-5408 we recall that the first term $\left(\Omega \times 2 m_{\text {Bub. }} r v_{\mathrm{A}}\right)$ in the torque equation gives a decreasing torque with radius while the second one $\left(\Omega \times \dot{m}_{\mathrm{Bub} .} r^{2}\right)$ gives an increasing torque with radius. Since the radius increases in time this is equivalent to the torque changing accordingly in time. In order for the torque to increase with time then $\dot{m}_{\mathrm{Bub}} r^{2}>2 m_{\mathrm{Bub}} r v_{\mathrm{A}}$ which at a distance half way to the 1.c. implies $\dot{m}_{\text {Bub. }} / m_{\text {Bub. }}>10^{-5} / P^{7 / 2}$. Thus a slight increase in the bubble's mass during propagation can give an increasing torque with time.

\section{Discussion}

\subsection{Transient versus normal AXPs during quiescence in our model}

The two sources studied here are termed transients in the sense that in quiescence their measured temperatures are as low as those of some ordinary young neutron stars. Only during their bursting phase that their bolometric luminosity becomes comparable to that of a typical AXP in quiescence (i.e. a bolometric luminosity of $\sim 10^{35} \mathrm{erg} \mathrm{s}^{-1}$; see Fig. 2 in OLNIII). From approximate outburst time, the estimated initial bolometric luminosities for the two transients are $\sim 2 \times 10^{35} d_{3.3}^{2} \mathrm{erg} \mathrm{s}^{-1}$.

In our model, to understand the difference between transient and normal AXPs during quiescence, one should note that the magnetic field would impose co-rotation of the ring's atmosphere (thus no accretion onto the star) as long as the magnetic energy density anywhere along the ring's atmosphere exceeds the Keplerian energy density. The condition is

$\zeta=\frac{\rho_{\mathrm{atm}} v_{\mathrm{K}}^{2}}{B^{2} / 8 \pi} \simeq 0.2 \frac{T_{\mathrm{keV}}^{3 / 2} M_{1.4} R_{\mathrm{out}, 25}^{5}}{B_{\mathrm{s}, 14}^{2} R_{\mathrm{QS}, 10}^{6}}<1$. 
Thus during the evolution of the source as long as the condition above is satisfied there will be no accretion and the object's emission is dominated by vortex expulsion.

The star's magnetic field decays at a rate (see Sect. 3 in Niebergal et al. 2006)

$$
B_{\mathrm{s}}=B_{0}\left(1+\frac{t}{T}\right)^{1 / 6}
$$

where $T=840 \mathrm{~s}$ is the characteristic age due to vortex expulsion using our fiducial value of $B_{\mathrm{QS}, 0}=10^{15} \mathrm{G}$ and $P_{\mathrm{QS}, 0}=1 \mathrm{~ms}$ for the star's surface magnetic field and period at birth, respectively. Simultaneously, the ring spreads viscously outward (i.e. radially) at a rate given by Eq. (A.7) in OLNII

$$
(\Delta r)_{\text {ring }} \simeq 7.8 \mathrm{~km} T_{\mathrm{keV}}^{5 / 4} t_{\mathrm{yr}}^{1 / 2},
$$

where time is in years. The ring's radius at birth is given by Eq. (2) in OLNII and is $R_{\text {out }, 0} \simeq 15 \mathrm{~km} / m_{-7}^{2}$ where $m_{-7}$ is the ring's mass is in units of $10^{-7} M_{\odot}$ so that it spreads from $R_{\text {out }, 0}$ to $R_{\text {out }}=R_{\text {out }, 0}+(\Delta r)_{\text {ring }}$; clearly for sources thousands of years old (as is the case here) the ring's outer radius is simply given by $R_{\text {out }} \sim(\Delta r)_{\text {ring }}$. The condition for no accretion, $\zeta<1$, is then equivalent to

$t<1300 \mathrm{yrs} \times T_{\mathrm{keV}}^{-93 / 26} \times B_{0,15}^{12 / 13}$.

To get Eq. (24) we start with condition (21) and $R_{\text {out }}=\Delta r_{\text {ring }}$ (i.e. Eq. (23)) and replaced $B_{\mathrm{s}}$ from Eq. (22) with $t \gg T$; The QS mass and radius are kept to their fiducial values. This implies that for $B_{0}=10^{15} \mathrm{G}$ and $0.2<T(\mathrm{keV})<0.4$, the transient age is $3 \times 10^{4}<t(\mathrm{yrs})<4 \times 10^{5}$. Transient AXPs, we speculate, should be younger than accretion-dominated sources (such as 1E2259+586 and 4U0142+61) in our model.

The magnetic field decay from vortex expulsion implies that magnetic field lines deflate radially inwards ${ }^{3}$, so we expect that violation of co-rotation to first occur at the ring's outer edge. Also, at the outer edge of the disk, there is magnetic field of opposite $B_{\phi}$ being produced when matter drags field lines. This is an unstable situation since reconnection can occur between adjacent field lines of opposite polarity leading to accretion along the reconnecting field lines. Anywhere else on the surface of the disk, whenever $\zeta>1$, the atmosphere winds up the field lines without reconnecting them until the magnitude of the magnetic field is strong enough to re-inforce corotation, i.e. the magnetic field stiffens, leading to a stable balance.

In summary, a transient AXP quiescent phase is dominated by $\mathrm{X}$-ray emission from vortex expulsion only $\left(L_{\mathrm{X} \text {,vortex }} \simeq 2 \times\right.$ $10^{34} \dot{P}_{-11} \mathrm{erg} \mathrm{s}^{-1}$ ) with the source continuing to evolve along the vortex band (see Fig. 2 in OLNIII). On the other hand, a typical AXP (older ring-bearing source) quiescent phase is dominated by emission from the HS induced by accretion from the outer

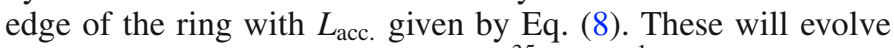
horizontally (at constant $L_{\mathrm{HS}} \sim 10^{35} \mathrm{erg} \mathrm{s}^{-1}$ ) as discussed in OLNII (see Fig. 2 in OLNIII).

It is only during the bursting phase that transient and regular AXPs would look the same since they both experience the $\mathrm{BF}$ effect and the related accretion and feedback process. In the transient case, as the BF dies out when reaching the outer edge, the system becomes dominated again by vortex expulsion while regular AXPs resume their accretion dominated (from the outer edge) quiescent phase.

3 Inside the star, the field lines expand outwards following the vortices.
Table 1. Thermal components in our model.

\begin{tabular}{clc}
\hline \hline Sources & Quiescent phase & Bursting phase \\
\hline SGRs & $2 \mathrm{BBs}$ & $3 \mathrm{BBs}$ \\
Transient AXPs & $2 \mathrm{BBs}$ & $3 \mathrm{BBs}$ \\
Typical AXPs & $3 \mathrm{BBs}$ & $3 \mathrm{BBs}$ \\
\hline
\end{tabular}

\subsection{Birthrate}

In our model, a QS-ring system experiences an X-ray/radio outburst every few hundred years. Since we have observed 2 in a few years, located at distances of 3 to $9 \mathrm{kpc}$ away (meaning we see about $30 \%$ of them), it is suggestive of a rate of 1 per year for the whole galaxy. This implies a total population of $(1 / \mathrm{yr}) \times 100 \mathrm{yrs} \sim 100$ in the galaxy. Since the ring will be consumed on a timescale of $\sim 10^{4}-10^{5}$ years (see Eq. (5) in OLNIII) this gives a birthrate of (1 per 1000 years) to (1 per 100 years). Within uncertainties, the birth rate of transient AXPs derived above is consistent with the expected birthrate of AXPs ( 1/300 yrs; Gill \& Heyl 2007; Leahy \& Ouyed 2009). As we argued in the previous section, in our model transient AXPs evolve into typical AXPs thus sharing the same birthrate.

\subsection{Model features and predictions}

The general predictions, starting with the X-ray emission, in our model are:

- The 2 blackbodies: overall, during burst and quiescence, the cool BB (from Keplerian ring; or from the co-rotating shell as in OLNI) arises from reprocessing radiation of the hot BB from the star (either vortex annihilation or accretion HS). We suggest, the relations in Fig. 6 in Nakagawa et al. (2009) can be explained in the context of reprocessing during both quiescence and burst. Table 1, summarizes the different emission components for different objects in different states in our model. Compared to SGRs (see OLNI) and transient AXPs, typical AXPs acquire an additional BB from the HS during quiecence. Only during bursting do transient AXPs acquire a HS. As for SGRs in our model, we recall (see OLNI) they are born with a co-rotating shell (i.e. non-Keplerian degenerate ring; see OLNI). Future work will consider hard X-ray emission from non-thermal processes related to magnetic reconnection following vortex expulsion and to accretion onto the QS.

- During $X$-ray burst: since the accreted material in our model consists mostly of dissociated iron $(Z \sim 13)$, we predict some sort of signatures either during channeling along the field line or on impact on the HS - maybe absorption lines or proton cyclotron lines $\left(\sim 0.14 B_{\mathrm{QS}, 14} \mathrm{keV}\right)$ from any of element in the Ne-to-S group (e.g. Ne, Al, Si). These signatures should be common for both transient and typical AXPs in our model.

- During X-ray quiescence: when edge accretion occurs the signatures (e.g. absorption lines or proton cyclotron lines) should be from the $Z=26$ rather than the $Z \sim 13$ nuclei.

- X-ray variability: during both the quiescent and bursting phases, X-rays from the ring+atmosphere system should be unpulsed and may carry the Keplerian sgnature via millisecond variability.

The general predictions for the radio emission are:

- Radio delay: as can be seen from Eq. (14), the shorter the period of the star the smaller the delay, $t_{\text {delay }}$, between the 
$\mathrm{X}$-ray burst and the radio emission. Interestingly, the 2 radio emitting AXPs so far are observed are those with the smallest period.

- Radio flux: furthermore, as can be seen from Eq. (16), as $P$ gets larger the radio following $X$-ray burst gets very faint making it more difficult to detect. Combined with long $t_{\text {delay }}$ for large $P$, we argue these to be the reasons why radio is not observed following X-ray bursts in AXPs with higher $(\sim 10 \mathrm{~s})$ period.

Finally, we list specific predictions in our model in the case of XTE J1810-197:

- XMM warm BB: the XMM warm BB for XTE J1810-197 (ring+atmosphere system in our model) will evolve back to one single BB (ROSAT BB) with temperature $T_{\mathrm{q}}$.

- XMM hard BB: the XMM hard BB (the accretion HS in our model) will disappear following burst (i.e. once the Bohm front reaches $R_{\text {out }}$ ). However, we expect edge effects to appear (e.g. flattening or even jump in the hot BB area; see last panel in Fig. 3) just before accretion shuts off.

\subsection{Further implications}

There exist two aspects of our model that might provide some answers to fundamental issues in pulsar magnetospheres ${ }^{4}$. First, the source in the quark-nova model is born as an aligned rotator and secondly is the fact that here the degenerate ring, by Keplerian shear, is a natural source of plasma (carried by the bubbles to the 1.c.) for the magnetosphere. Also, pairs are naturally supplied to the magnetosphere by vortex annihilation. This is left as an avenue for future investigation.

\subsection{Model Limitations}

Our model suffers from a few caveats :

(i) the interaction between the $\mathrm{BF}$ and the magnetic field in the atmosphere is rudimentary at this stage. Understanding the exact mechanism of feeding material from degenerate ring into the atmosphere and onto the field lines within the Bohm front is essential as this process is behind the feedback process between the ring and the HS on the star. This is a complex problem/system that would require detailed MHD simulations before we can confirm this aspect of our model;

(ii) while the bubble generation mechanism we propose is common in systems involving dipole files threaded by a disk on one end and a star on the other, the outward propagation of the bubbles within the 1.c. depends crucially on conditions within the magnetosphere. In particular, the value $\alpha=1$ was chosen solely on the assumption that the bubbles will propagate in an equatorial wind (given the Keplerian nature of the bubbles at birth) within the magnetosphere. For highly magnetized, aligned rotators, the physics of the magnetosphere within the 1.c. is yet to be understood and the problem solved. Furthermore, the bubble dissipation mechanism

\footnotetext{
4 A disk model for X-ray emission from pulsars was considered by Michel \& Dessler (1981). However, their disk is fundamentally different from our ring in that, where they hypothesize electron degenerate material (left over from a supernova), in our model the material is completely relativistic-degenerate (from a Quark-Nova). This difference has many consequences, the most notable are a more efficient accretion mechanism (conversion energy from the hadron to quark transition as well as gravitational energy is released), and a slower (viscous) spreading rate for the ring.
}

as they reach the 1.c. remains to be demonstrated. The detailed structure of the thin shear layer at the light cylinder is not well studied and would require numerical simulations. For now our explanation is based on the assumption that the thin transition shear layer at the 1.c. slowly destroys the bubble by shearing off the part that touched the light cylinder.

\section{Conclusion}

There are two fundamental components in our model for AXPs and transient AXPs namely, the QS and the Keplerian ring. In quiescence, vortex annihilation on the QS gives rise to thermal and non-thermal X-ray emission. The ring reprocesses the emission to give a second cooler BB emission component. Outburst is triggered by accretion of a small inner part of the ring (i.e. the wall). The two main consequences are production of light $(Z \sim 13)$ nuclei and triggering MHD accretion onto the QS (yielding the HS). The interplay between the Bohm diffusion (i.e. $R_{\mathrm{BF}}$ term) and depletion of light nuclei (i.e. $\mu$ ) gives rise to a rich behavior, necessary in order to account for the observed behavior of XTE J1810-197. Finally, one can ask if such a small Keplerian degenerate iron-rich ring could form around a neutron star. Ring formation when the neutron star is born appears implausible since a proto-neutron star is large compared to the ring size. After formation, there is no obvious mechanism to eject degenerate material unless a violent change of state, like a QN occurs.

Acknowledgements. This research is supported by grants from the Natural Science and Engineering Research Council of Canada (NSERC). We thank the referee for comments that helped improve this paper.

\section{Appendix A: The Bohm diffusion front}

The hot front propagates from the inner parts of the ring at a Bohm diffusion rate given by

$\Delta r=\psi_{\mathrm{BF}}\left(6.25 \times 10^{-4} \frac{T_{\mathrm{kev}}}{B_{\perp, 13}}\right)^{1 / 2} t^{1 / 2}$,

where $B_{\perp, 13}$ is the magnetic field component perpendicular to the front motion in units of $10^{13} \mathrm{G}$. The parameter $\psi_{\mathrm{BF}}$ carries some uncertainty related to the fact that the above formula is semiempirical (i.e. the constant $1 / 16$ in the original Bohm diffusion expression has no theoretical justification; see Sect. 5.10 in Chen 1984). This parameter is derived self-consistently in our model as explained in Sect. B. 2 below. For a qualitative derivation of Bohm diffusion we refer the interested reader to Alexeff \& Rader (1991) and Belyaev (2001).

The ring geometry allows us to write $B_{\perp, 13} \simeq \tan \left(\alpha_{\mathrm{c}}\right) B_{\mathrm{BF}, 13}$ where to a first approximation $\tan \left(\alpha_{\mathrm{c}}\right)=\left(H_{\mathrm{atm} ., \text { out }}^{z}-\right.$ $\left.H_{\mathrm{atm} . \text { in }}^{z}\right) /\left(R_{\mathrm{out}}-R_{\mathrm{in}}\right)$ and $B_{\mathrm{BF}} \simeq B_{\mathrm{av} .}=B_{\mathrm{QS}}\left(R_{\mathrm{QS}} / R_{\mathrm{av} .}\right)^{3}$ where $R_{\mathrm{av} .}=\left(R_{\mathrm{in}}+R_{\text {out }}\right) / 2$; here $B_{\mathrm{QS}}=\sqrt{3 \kappa P \dot{P}}$ and $R_{\mathrm{QS}}$ are the star's magnetic field and radius, respectively. The temperature cancels out from Eq. (A.1) since $B_{\perp} \propto H_{\mathrm{atm}}^{z} \propto T_{\mathrm{keV}} / \mu$ so that,

$\Delta r \sim 10^{-2} \mathrm{~km} \psi_{\mathrm{BF}}\left(\frac{\mu_{3.3}}{B_{\mathrm{av} ., 13} R_{\mathrm{out}, 15}^{1 / 2}}\right)^{1 / 2} t_{\mathrm{days}}^{1 / 2}$.

\section{Appendix B: Centrifugal ejection and channeled accretion}

As the system (ring+atmosphere) is heated, the boundary between the non-degenerate atmosphere and the degenerate 
$Z \sim 13$ layer moves downwards into higher density layers (see Eq. (1)). Thus Keplerian ring material is fed into the atmosphere and is ejected as a magnetohydrodynamic (MHD) wind as outlined below (see also lower panel in Fig. 2).

At $\mathrm{keV}$ temperatures the gas is sufficiently ionized everywhere in the atmosphere that ideal MHD can be used. Since the Lorentz force only has components perpendicular to the field, the gas is free to move along the co-rotating field line under the influence of other forces. Under these conditions, it has been shown that the wind can be launched centrifugally if the field direction is inclined at an angle less than $60^{\circ}$ to the radial direction (Blandford \& Payne 1982). Using conservation of specific angular momentum $\left(\propto r^{2} \Omega\right)$, a non-degenerate Keplerian particle (i.e. $\Omega_{\mathrm{K}}$ ) loaded at a footpoint $r_{0}$ will be flung out to larger radii; the so-called "bead-on-wire" analogy. These conditions are easily met in our model so that the newly unveiled Keplerian material (following heating of the degenerate ring) finds itself threaded by the highly inclined magnetic field and is flung out centrifugally. The wind is then channeled towards the star's surface by the strong dipole.

The mass flux, $\dot{m}_{\text {wind }}$, is regulated by conditions (i.e. density) at the slow mode critical point:

$\dot{m}_{\mathrm{acc}}=4 \pi R_{\mathrm{BF}} \zeta_{\mathrm{sp}}\left(\rho_{\mathrm{atm} . \mathrm{b}} H_{\mathrm{atm} . \mathrm{b}}^{z}-\rho_{\mathrm{atm} ., \mathrm{q}} H_{\mathrm{atm} ., \mathrm{q}}^{z}\right) v_{\mathrm{atm}, \mathrm{th}}$,

where $\left(\rho_{\text {atm.,b }} H_{\text {atm.,b }}-\rho_{\text {atm.,q }} H_{\text {atm., }}\right)=\rho_{\text {atm.,b }} H_{\text {atm.,b }} \times f_{\mathrm{K}}\left(T_{\mathrm{b}}\right)$ describes the mass injected from the Keplerian ring into the atmosphere. The function $f_{\mathrm{K}}\left(T_{\mathrm{b}}\right)=1-T_{\mathrm{q}}^{5 / 2} / T_{\mathrm{b}}^{5 / 2}$ evolves from $\sim 0.8$ to zero as the non-degenerate Keplerian material is depleted and the system evolves back to its quiescent phase. In a sense it acts as a "valve" that shuts-off accretion after the heating front has passed.

The accretion rate, $\dot{m}_{\text {acc. }} \sim \dot{m}_{\text {wind }}$, can be recast to

$\dot{m}_{\text {acc. }}=1.77 \times 10^{17} \zeta_{\mathrm{sp}, 0.01} \frac{T_{\mathrm{b}, \mathrm{keV}}^{3} f_{\mathrm{K}}\left(T_{\mathrm{b}}\right) R_{\mathrm{BF}, 15}^{5 / 2}}{\mu^{3 / 2}}$.

The factor $\zeta_{\text {sp }}$ carries the uncertainty on the exact location of the slow MHD mode. We will adopt $\zeta_{\mathrm{sp}} \sim 0.01$ as inferred from 2-dimensional (Ouyed et al. 1997) and 3-dimensional (Ouyed et al. 2003) MHD simulations of disk winds. The slow MHD point governs the mass loss rate. The degenerate ring responds by suppling mass at a rate $\dot{m}_{\mathrm{BF}} \sim \rho_{\mathrm{atm}} 2 \pi R_{\mathrm{BF}} d_{\mathrm{BF}} v_{\perp}$ where $v_{\perp}$ is the velocity of degenerate Keplerian material crossing the boundary to non-degeneracy ${ }^{5}$, and $d_{\mathrm{BF}}$ is the radial width of the $\mathrm{BF}$ as illustrated in Fig. 2.

\section{B.1. Accretion shut-off}

The accretion relies upon new Keplerian material being fed from the degenerate ring to the atmosphere in the $\mathrm{BF}$ region, and ends once the magnetic field has re-inforced co-rotation of the atmosphere. This defines the inner edge of the BF as illustrated in the lower panel of Fig. 2. The penetration timescale can be estimated from Eq. (16) in OLNII to be $\tau_{\mathrm{B}, \mathrm{K}} \sim 4$ days $H_{\mathrm{K}, \mathrm{cm}}^{2} / T_{\mathrm{keV}}^{1 / 2}$ where the depth of the non-degenerate Keplerian layer $H_{\mathrm{K}} \sim$ $10^{-3} H_{13} \sim 10^{-6} H_{\text {ring }}$ is in units of centimeters. The size (i.e. radial width) of the BF front is found from $d_{\mathrm{BF}}=v_{\mathrm{BF}} \tau_{\mathrm{B}, \mathrm{K}} \sim 10^{3} \mathrm{~cm}$ which is of the same order as the depth of the $Z \sim 13$ layer.

\footnotetext{
5 We recall that in the degenerate Keplerian disk, lower viscosity and conservation of angular momentum imply mass flows in the ring is along lines of constant angular momentum which is nearly vertical (i.e. $z$-direction; see Appendix A in OLNII). Thus as the non-degenerate Keplerian material is "sucked up" in the wind, more material is supplied almost vertically from the underlying Keplerian material.
}

\section{B.2. Depletion timescale of the $Z \sim 13$ nuclei}

We recall that following wall accretion and subsequent irradiation of the ring, up to $10^{46}$ of iron nuclei are dissociated forming the $Z \sim 13$ layer. Subsequent depletion of the $Z \sim 13$ nuclei by accretion leads to a return to an iron-rich atmosphere (i.e. $\left.\mu_{\mathrm{q}} \sim 3.3\right)$ as shown in OLNII:

$\frac{1}{\mu}=\frac{1}{\mu_{\mathrm{q}}}+\left(\frac{1}{\mu_{\mathrm{b}}}-\frac{1}{\mu_{\mathrm{q}}}\right) \exp ^{-t / \tau_{13}}$,

where $\tau_{13}$ is the depletion timescale given by $\tau_{13} \sim m_{13} / \dot{m}_{\text {acc. }}$ where $m_{13} \sim N_{13} \times\left(13 m_{\mathrm{H}}\right)$. For typical values following wall accretion $\mu=\mu_{\mathrm{b}} \sim 2.3, R_{\mathrm{BF}} \sim R_{\mathrm{in}}, Y_{\mathrm{BF}} \sim 0.4$, and $\eta=0.1$, we find

$\tau_{13} \sim 1.4 \times 10^{4}$ days $\frac{N_{13,46}}{\zeta_{\mathrm{sp}, 0.01}^{4}}$,

where $N_{13,46}$ is the total number of dissociated iron nuclei in units of $10^{46}$.

We have already estimated the size of the BF region above which we found to be of the same order as the depth of the $Z \sim 13$ layer which implies that our scenario self-consistently leads to the accretion of most of the $Z \sim 13$ material during burst (i.e. during the time it takes the BF to comb the ring's surface). In other words, the depletion timescale can also be estimated from Eq. (A.2) by taking $\Delta r=\left(R_{\text {out }}-R_{\text {in }}\right)$ and $\mu=\mu_{\mathrm{b}} \sim 2.3$ to get

$\tau_{13} \sim \frac{1.5 \times 10^{4}}{\psi_{\mathrm{BF}}^{2}}$ days $\left(R_{\mathrm{out}}-R_{\mathrm{in}}\right)_{\mathrm{km}}^{2} R_{\mathrm{out}, 15}^{1 / 2} B_{\mathrm{av} ., 13}$,

where $\left(R_{\text {out }}-R_{\text {in }}\right)$ is in units of kilometers. Equating the previous two equations we find

$\psi_{\mathrm{BF}} \sim \frac{\zeta_{\mathrm{sp}, 0.01}^{2}\left(R_{\mathrm{out}}-R_{\mathrm{in}}\right)_{\mathrm{km}} R_{\mathrm{out}, 15}^{1 / 4} B_{\mathrm{av} ., 13}^{1 / 2}}{N_{13,46}^{1 / 2}}$,

which fine tunes the speed of the BF in our model (i.e. Eq. (A.2)).

\section{Appendix C: Ring-Pole interaction: the hot spot}

As illustrated in Fig. 2, there is a direct link between the BF moving outward along the ring surface (increasing its area) while decreasing the area of the HS on the surface of the star. It is straightforward to show that the total HS area (both poles) is

$A_{\mathrm{HS}}=4 \pi R_{\mathrm{QS}}^{2} \times\left(\cos \beta_{\mathrm{BF}}-\cos \beta_{\mathrm{in}}\right)$,

where the angle $\beta$ defines the colatitude (i.e. measured from the polar axis) on the star's surface where material channeled along a given field line lands. We approximate the field by a dipole configuration, giving us

$\sin \beta=\left(\frac{R_{\mathrm{QS}}}{R}\right)^{1 / 2} \sin \alpha$,

where

$\alpha=\frac{\pi}{2}-\arctan \frac{H_{\text {ring }}}{R}$,

is the angle from the polar axis to the footpoint, at radial distance $R$, of the field line where the Keplerian material is loaded; $H_{\text {ring }} \sim 2.68 \mathrm{~km} \rho_{\text {ring, } 9}^{1 / 6} R_{15}^{3 / 2}$ is the ring's vertical height as given in Sect. 2.2. For our fiducial values, $R_{\text {in }}=15 \mathrm{~km}, R_{\text {out }}=25 \mathrm{~km}$ and $R_{\mathrm{QS}}=10 \mathrm{~km}$, we find $\left(\cos \beta_{\text {out }}-\cos \beta_{\text {in }}\right) \sim 0.192$ which gives an initial area of the HS at one pole of the order of $\sim 120 \mathrm{~km}^{2}$. 
R. Ouyed et al.: Quark-nova and radio-emitting AXP transients. IV.

\section{References}

Alexeff, I., \& Rader, M. 1991, A simple derivation of classical and Bohm diffusion based on magneto-hydro-dynamics, in Proc. Conf. Rec. IEEE Int. Conf. Plasma Sci., Williamsburg, VA, Jun. 35, 199

Belyaev, V. 2001, Anomalous diffusion and photon-field fusion, 5th workshop on fast ignition of fusion targets, Funchak, 18022 June, 18

Bernardini, F., Israel, G. L., Dall'Osso, S., et al. 2009, A\&A, 498, 195

Blandford, R. D., \& Payne, D. G. 1982, MNRAS, 199, 883

Camilo, F., Ransom, S. M., Halpern, J. P., et al. 2006, Nature, 442, 892

Camilo, F., Ransom, S. M., Halpern, J. P., \& Reynolds, J. 2007a, ApJ, 666, L93

Camilo, F., Cognard, I., Ransom, S. M., et al. 2007b, ApJ, 663, 497

Camilo, F., Ransom, S. M., Peñalver, J., et al. 2007c, ApJ, 669, 561

Camilo, F., Reynolds, J., Johnston, S., Halpern, J. P., \& Ransom, S. M. 2008, ApJ, 679, 681

Camilo, F., Halpern, J. P., \& Ransom, S. M. 2009, The Astronomer's Telegram, 1907, 1

Chen, F. F. 1984, Introduction to Plasma Physics and Controlled Fusion (Springer)

Gelfand, J. D., \& Gaensler, B. M. 2007, ApJ, 667, 1111

Gill, R., \& Heyl, J. 2007, MNRAS, 381, 52

Gotthelf, E. V., \& Halpern, J. P. 2005, ApJ, 632, 1075

Gotthelf, E. V., \& Halpern, J. P. 2007, Ap\&SS, 308, 79

Halpern, J. P., \& Gotthelf, E. V. 2005, ApJ, 618, 874

Halpern, J. P., Gotthelf, E. V., Reynolds, J., Ransom, S. M., \& Camilo, F. 2008 , ApJ, 676, 1178

Ibrahim, A. I., Markwardt, C. B., Swank, J. H., et al. ApJ, 2004, 609, L21

Jaroschek, C. H., Lesch, H., \& Treumann, R. A. 2004, ApJ, 605, L9

Kaspi, V. M. 2007, Ap\&SS, 308, 1

Keränen, P., \& Ouyed, R. 2003, A\&A, 407, L51
Keränen, P., Ouyed, R., \& Jaikumar, P. 2005, ApJ, 618, 485

Lang, K. R. 1999, Astrophysical formulae, ed K. R. Lang (New York: Springer), Astronomy and Astrophysics Library, ISSN0941-7834

Leahy, D., \& Ouyed, R. 2009, Adv. Astron., 2009, 1

Leahy, D., \& Ouyed, R. 2008, MNRAS, 387, 1193

Michel, F. C., \& Dessler, A. J. 1981, ApJ, 251, 654

Michel, F. C. 1991, Theory of neutron star magnetospheres (Chicago, IL: University of Chicago Press)

Nakagawa, Y. E., Yoshida, A., Yamaoka, K., \& Shibazaki, N. 2009, PASJ, 61, 109

Niebergal, B., Ouyed, R., \& Leahy, D. 2006, ApJ, 646, L17

Ouyed, R., \& Leahy, D. 2009, ApJ, 696, 562

Ouyed, R., Pudritz, R. E., \& Stone, J. M. 1997, Nature, 385, 409

Ouyed, R., Dey, J., \& Dey, M. 2002, A\&A, 390, L39

Ouyed, R., Clarke, D. A., \& Pudritz, R. E. 2003, ApJ, 582, 292

Ouyed, R., Elgarøy, Ø., Dahle, H., \& Keränen, P. 2004, A\&A, 420, 1025

Ouyed, R., Niebergal, B., Dobler, W., \& Leahy, D. 2006, ApJ, 653, 558

Ouyed, R., Leahy, D., \& Niebergal, B. 2007a, A\&A, 473, 357 (OLNI)

Ouyed, R., Leahy, D., \& Niebergal, B. 2007b, A\&A, 475, 63 (OLNII)

Ouyed, R., Leahy, D., Niebergal, B., \& Yue, Y. 2009, MNRAS, 396, 1058 (OLNIII)

Perna, R., \& Gotthelf, E. V. 2008, ApJ, 681, 522

Rajagopal, K., \& Wilczek, F. 2001, Phys. Rev. Lett., 86, 3492

Romanova, M. M., Ustyugova, G. V., Koldoba, A. V., Chechetkin, V. M., Lovelace, R. V. E., et al. 1998, ApJ, 500, 703

Spitkovsky, A. 2008, 40 Years of Pulsars: Millisecond Pulsars, Magnetars and More, 983, 20

Tamano, T. 1991, Sol. Phys., 134, 187

Wagner, W. J. 1984, Ann. Rev. Astr. Ap., 22, 267

Woods, P. M., \& Thompson, C. 2006, Compact stellar X-ray sources, 547

Yelenina, T. G., Ustyyugova, G. V., \& Koldoba, A. V. 2006, A\&A, 458, 679 